\title{
REMARKS ON CLASSICAL INVARIANT THEORY
}

\author{
ROGER HOWE
}

\begin{abstract}
A uniform formulation, applying to all classical groups simultaneously, of the First Fundamental Theory of Classical Invariant Theory is given in terms of the Weyl algebra. The formulation also allows skew-symmetric as well as symmetric variables. Examples illustrate the scope of this formulation.
\end{abstract}

\section{INTRODUCTION}

(N.B. This introductory discussion is somewhat breezy. We will be more careful beginning in $\S 1$.)

In Hermann Weyl's wonderful and terrible ${ }^{1}$ book, The classical groups [W], one may discern two main themes: first, the study of the polynomial invariants for an arbitrary number of (contravariant or covariant) variables for a standard classical group action; second, the isotypic decomposition of the full tensor algebra for such an action. It may be observed that the two questions are more or less equivalent. (Indeed, Weyl exploits one direction of this equivalence.) Further, it is not hard to see that both questions are equivalent to an apparently simpler one, namely the description of the invariants in the full (mixed) tensor algebra of such an action. (These equivalences are not a priori clear. It is the cleanness of the answers that allow one to make the connections.) Thus if Weyl had first established the result on tensor invariants, then proceeded to the other two topics, his presentation would have been considerably streamlined and presumably more acceptable to modern taste. A plausible explanation for his failure to proceed along that route is that he saw no way of establishing the result on tensor invariants except through the result on polynomial invariants. To produce the latter, he had the machinery of 19th century invariant theory available, in particular "the mysterious Capelli identity" (see [ABP, p. 324). This, together with the fact that polynomials can be thought of as functions,

Received by the editors March 8, 1989.

1980 Mathematics Subject Classification (1985 Revision). Primary 15A72, 20G05; Secondary 20C35, 20G45, 22E45, 22E70, 81C40.

Key words and phrases. Invariant theory, Weyl algebra, spherical harmonics.

Editor's note. This paper was written in 1976 and has been widely circulated as a preprint. There have been many developments related to the work since that time. However, because of its influence and historical value, the paper is being published here in the original form.

${ }^{1}$ Most people who know the book feel the material in it is wonderful. Many also feel the presentation is terrible. (The auihor is not among these latter.) 
hence are more tangible than general tensors, perhaps led Weyl to choose the path he did.

There was one case, however, namely for $\mathrm{Gl}_{n}$, in which the theorem on tensor invariants was directly available, by a beautiful argument (due to Schur) using the double commutant theorem. Weyl did not neglect to present this; in fact, it was a favorite of his. In itself, however, it would not serve as a basis for the general case, so could only be presented for its intrinsic merits, and led to greater heterogeneity in the book instead of unity. Recently, however, it was shown in [ABP] how the result on tensor invariants for orthogonal groups $\mathrm{O}_{n}$ could be reduced by a fairly quick argument to the result for $\mathrm{Gl}_{n}$. A similar argument goes through for the symplectic groups $\mathrm{Sp}_{2 n}$. Thus the description of tensor invariants for a standard classical action may now be considered to be available from first principles, and may be used to provide an efficient and unified treatment of a large portion of the material in [W].

The main purpose of the present discussion is to point out that this unified viewpoint is in fact capable of considerable extension. We note the following specifics.

(a) The argument used to pass from tensor invariants to polynomial (e.g., symmetric tensor) invariants in many variables applies equally well to the description of mixed algebras of partially symmetric, partially antisymmetric tensors.

(b) By means of a process of "doubling the variables", the result in (a) yields a description of the endomorphisms (of a certain reasonable type) commuting with a classical action on such an algebra.

(c) The result of (b) may be viewed as a "duality theorem" for commuting subalgebras of a certain naturally occurring graded Lie algebra.

(d) Particular cases of the above general scheme yield many classical computations in multilinear algebra, often with additional structure and insight. We will give as examples the following:

(i) the full isotypic decomposition of the polynomials in $k$ variables for a classical action (after the archetypal case, this may be referred to as the theory of spherical harmonics);

(ii) the exactness of the polynomial de Rham and Dolbeault complexes, and the homotopy between the identity and projection onto the constants (used as in $[\mathrm{J}]$ to prove the Hilbert Syzygy Theorem);

(iii) the algebra behind the structure of the cohomology of Kähler manifolds (sometimes called Hodge Theory) as a special case of the analogue of (i) for exterior algebras (see [Wi]);

(iv) the cohomology of the unitary group;

(v) the invariants of the adjoint action of the classical groups;

(vi) elucidation of the Capelli identity.

These computations are not usually mentioned in the same breath and certainly are not all considered as part of classical invariant theory (though discerning eyes have perhaps noticed connections). Thus it seems fair to say that 
the result on tensor invariants forms the basis for a unified development of a considerable tract of algebra. Perhaps not least important, the results on duality make precise in a strong way the striking analogy, remarked on by a number of authors, between the Clifford algebra and the spin representation on the one hand and the Weyl algebra and the oscillator or (Segal-Shale) Weil or metaplectic or... representation on the other (or as physicists would say, the canonical anticommutation relations and canonical commutation relations).

This matter should emerge clearly in the body of the paper.

\section{INVARIANTS}

We will always work over $\mathbf{C}$, the complex numbers. Let $V$ be a complex vector space, and let $\langle$,$\rangle be a bilinear form on V$. We call the pair $(V,\langle\rangle$, a formed space. Sometimes we may speak of the formed space $V$, with $\langle$, being implicit. The group of isometries of $(V,\langle\rangle$,$) will be generally denoted$ by $G(V,\langle\rangle$,$) . There are three main cases we wish to consider and each has$ its particular symbol.

(a) If $\langle$,$\rangle is trivial, then G(V,\langle\rangle)=,\mathrm{GL}(V)$ is the general linear group of $V$, consisting of all invertible linear transformations of $V$.

(b) If $\langle$,$\rangle is nondegenerate and symmetric, then G(V,\langle\rangle$,$) is called an$ orthogonal group and denoted $\mathrm{O}=\mathrm{O}(V,\langle\rangle$,$) .$

(c) If $\langle$,$\rangle is nondegenerate and skew symmetric, then G(V,\langle\rangle$,$) is called$ a symplectic group and denoted $\mathrm{Sp}=\mathrm{Sp}(V,\langle\rangle$,$) .$

The isomorphism classes of these groups depend only on $n=\operatorname{dim} V$, so we will sometimes write $\mathrm{GL}(V)=\mathrm{GL}_{n}$. Similarly $\mathrm{O}(V,\langle\rangle)=,\mathrm{O}_{n}$. For $\operatorname{Sp}(V,\langle\rangle$,$) to exist, we must have n$ even: $n=2 m$. Thus we usually write $\mathrm{Sp}(V,\langle\rangle)=,\mathrm{Sp}_{2 m}$. The groups $\mathrm{GL}_{n}, \mathrm{O}_{n}$, and $\mathrm{Sp}_{2 m}$ are referred to collectively as the classical groups (over $C$ ). The action of a classical $G(V,\langle$,$\rangle ) on$ $V$ is called a standard classical action, and $V$ is the standard or basic module for $G$.

The first thing to do is to describe the tensor invariants of a standard classical action. The case of $\mathrm{GL}_{n}$ differs slightly from those of $\mathrm{O}_{n}$ and $\mathrm{Sp}_{2 m}$, and we deal with it first.

Let $V^{*}$ be the vector space dual of $V$. We let $\operatorname{GL}(V)$ act on $V^{*}$ in the usual contragredient manner, so as to preserve the natural pairing between $V$ and $V^{*}$. Thus if $g \in \mathrm{GL}(V), v \in V$, and $v^{*} \in V^{*}$, then we define $g\left(v^{*}\right)$ by the formula

$$
g\left(v^{*}\right)(v)=v^{*}\left(g^{-1}(v)\right)
$$

Consider a tensor product $V_{k l}=\left(\otimes^{k} V\right) \otimes\left(\otimes^{l} V^{*}\right)$ made from $k$ copies of $V$ and $l$ copies of $V^{*}$. We let $\operatorname{GL}(V)$ act on this by the linear extension of the obvious formula

$$
\begin{aligned}
g\left(v_{1}\right. & \left.\otimes v_{2} \otimes \cdots \otimes v_{k} \otimes v_{1}^{*} \otimes \cdots \otimes v_{l}^{*}\right) \\
& =g\left(v_{1}\right) \otimes g\left(v_{2}\right) \otimes \cdots \otimes g\left(v_{k}\right) \otimes g\left(v_{1}^{*}\right) \otimes \cdots \otimes g\left(v_{l}^{*}\right) .
\end{aligned}
$$


It is clear that there can be no invariants for this action unless $k=l$ since if $g=\alpha 1_{V}$, where $1_{V}$ is the identity on $V$ and $\alpha \in \mathbf{C}$, then $g(t)=\alpha^{k-l} t$ for any $t \in V_{k l}$. Thus assume $k=l$. Consider the canonical identification $i_{1}: V_{11} \simeq$ $\operatorname{End}(V)$, where $\operatorname{End}(V)$ is the algebra of linear transformations on $V$. This gives rise to an identification $i_{k}: V_{k k} \simeq \bigotimes^{k} \operatorname{End}(V)$ of $k$-fold tensor products. Let Ad be the action of $\mathrm{GL}(V)$ on $\operatorname{End}(V)$ by conjugation: $\operatorname{Ad} g(T)=g T g^{-1}$ for $g \in \mathrm{GL}(V)$ and $T \in \operatorname{End}(V)$. Then $i_{1}$ identifies the action of $\mathrm{GL}(V)$ on $V_{11}$ with Ad. That is

$$
i_{1}(g(t))=\operatorname{Ad} g\left(i_{1}(t)\right) \quad \text { for } t \in V_{11} .
$$

Thus $i_{1}^{-1}\left(1_{V}\right)$ is an invariant for $\operatorname{GL}(V)$ acting on $V_{11}$, and $\otimes^{k}\left(i_{1}^{-1}\left(1_{V}\right)\right)$, its $k$-fold tensor power, is an invariant for $\mathrm{GL}(V)$ acting on $V_{k k}$. Denote it by $\theta_{k}$.

Observe that we may permute among themselves the factors of $V_{k k}$ isomorphic to $V$. Similarly we may permute the factors of $V_{k k}$ isomorphic to $V^{*}$. Thus if $S_{k}$ is the symmetric group on $k$ letters we have an action of $S_{k} \times S_{k}$ on $V_{k k}$, and this action commutes with the action of $\operatorname{GL}(V)$ on $V_{k k}$. Thus the $\mathrm{GL}(V)$-invariants form a module for $S_{k} \times S_{k}$. We see that $\theta_{k}$ is invariant under the diagonal subgroup of $S_{k} \times S_{k}$.

Theorem 1A. Translates of $\theta_{k}$ by $S_{k} \times S_{k}$ span the $\mathrm{GL}(V)$-invariants in $V_{k k}$.

This is in Weyl [W]. Notice that $V_{k k} \simeq \operatorname{End}\left(V_{k 0}\right)$ and the action of $\mathrm{GL}(V)$ on $V_{k k}$ is just the conjugation action coming from its action on $V_{k 0}$. Thus the $\mathrm{GL}(V)$ invariants in $V_{k k}$ are just the endomorphisms of $V_{k 0}$ commuting with $\mathrm{GL}(V)$. Further $S_{k}$ acts on $V_{k 0}$, so $S_{k} \subseteq \operatorname{End}\left(V_{k 0}\right)$ and the above action of $S_{k} \times S_{k}$ on $V_{k k}$ corresponds to pre- and post-multiplication by $S_{k}$ in $\operatorname{End}\left(V_{k 0}\right)$. Also $\theta_{k}$ corresponds to the identity operator on $V_{k 0}$. Therefore Theorem $1 \mathrm{~A}$ says that permutations of the factors of $V_{k 0}$ yield all operators commuting with $\mathrm{GL}(V)$. This is the form of the result of Weyl.

Now pass to consideration of $\mathrm{O}_{n}$ and $\mathrm{Sp}_{2 m}$. Denote one of these by $G$ and let $V$ be the standard module for $G$, with form $\langle$,$\rangle . We have the$ isomorphism $\beta: V \rightarrow V$ given by

$$
\beta\left(v_{2}\right)\left(v_{1}\right)=\left\langle v_{1}, v_{2}\right\rangle
$$

The map $\beta$ commutes with the natural actions of $G$ on $V$ and on $V^{*}$, so these spaces define isomorphic $G$-modules. Thus here it is unnecessary to explicitly consider mixed tensors, a slight simplification compared with $\mathrm{GL}_{n}$. Therefore, consider $V_{k}=\otimes^{k} V$. Note that $\langle$,$\rangle forms an invariant for G$ in $V_{2}^{*}$ which we may identify with $V_{2}$. Call this invariant $\theta_{2}$. If $k$ is even, say $k=2 j$, then we may choose an isomorphism

$$
i_{k}: V_{k} \simeq\left(V_{2}\right)_{j}=\bigotimes^{j} V_{2}
$$

Specifying $i_{k}$ amounts to pairing off the factors of $V_{k}$. In $\left(V_{2}\right)_{j}$ we have the invariant $\otimes^{j} \theta_{2}$. Thus in $V_{k}$ we have the invariant $\theta_{k}=i_{k}^{-1}\left(\otimes^{j} \theta_{2}\right)$. On $V_{k}$ 
we again have the action of $S_{k}$ permuting the factors. We see $\theta_{k}$ is invariant up to sign by the subgroup of $S_{k}$ which preserves the pairing used to define $i_{k}$.

Theorem 1B. If $k$ is odd, there are no G-invariants in $V_{k}$. If $k$ is even, the translates of $\theta_{k}$ by $S_{k}$ span the G-invariants in $V_{k}$.

This may also be dug out of Weyl. As mentioned in the introduction, a fairly quick proof, using $1 \mathrm{~A}$, is given in [ABP] for $\mathrm{O}_{n}$. One may argue similarly for $\mathrm{Sp}_{2 m}$.

We wish to transfer the above results to the context of certain other algebras. We would like to remark that the basic objects to be defined here and in $\S 2$ have also been defined independently and more elegantly by Tilgner $[T]$ as $\mathbf{Z}_{2}$-graded objects. The reader with a refined taste is referred to his article for comparison.

Let $U$ and $W$ be two complex vector spaces. Let $\mathscr{A}(U, W)$ be the associative algebra with 1 generated by $U$ and $V$ subject to the conditions that (i) elements of $U$ commute with everything and (ii) elements of $W$ anticommute among themselves. It is not hard to see that

$$
\mathscr{A}(U, W) \simeq S(U) \otimes \Lambda(W),
$$

where $S$ indicates the symmetric algebra and $\Lambda$ the exterior algebra. We may give $\mathscr{A}$ a grading by using the sum of the gradings on $S(U)$ and $\Lambda(W)$, so $\mathscr{A}$ is a graded algebra.

Next, consider a classical group $G$ acting on its basic module $V$. Choose positive integers $k_{i}, l_{i}, i=1,2$, and put $U=\left(\oplus^{k_{1}} V\right) \oplus\left(\oplus^{l_{1}} V^{*}\right)$ and $W=$ $\left(\oplus^{k_{2}} V\right) \oplus\left(\bigoplus^{l_{2}} V^{*}\right)$. (For $G=\mathrm{O}_{n}$ or $\mathrm{Sp}_{2 m}$, the explicit inclusion of $V^{*}$ in $U$ and $W$ is unnecessary.) Both $U$ and $W$ may be regarded as $G$-modules in the obvious way. Therefore we have on $\mathscr{A}(U, V)$ a natural $G$-action by algebra automorphisms. The invariants of this action therefore form a subalgebra containing the identity. In the algebra of invariants, the elements of total order two are easy to describe. They are simply the span of the canonical pairings between the various copies of $V$ and $V^{*}$. The extension of the First Fundamental Theorem of Classical Invariant Theory to the present context may be stated as follows.

Theorem 2. Let $G$ be a classical group, and let $U$ and $W$ be G-modules formed by taking direct sums of the basic module for $G$ (and, if $G=\mathrm{Gl}_{n}$, of the contragradient module). Consider the resulting action on $\mathscr{A}(U, W)$. Then the algebra of G-invariants is generated by the invariants of degree 2.

Proof. If $U=V^{k_{1}} \oplus V^{* l_{1}}$ and $W=V^{k_{2}} \oplus V^{* l_{2}}$ as indicated above, then $\mathscr{A}(U, V)$ is isomorphic as a $G$-module to the tensor product

$$
\left(\otimes^{k_{1}} S(V)\right) \otimes\left(\otimes^{l_{1}} S\left(V^{*}\right)\right) \otimes\left(\otimes^{k_{2}} \Lambda(V)\right) \otimes\left(\otimes^{l_{2}} \Lambda\left(V^{*}\right)\right) .
$$

Each factor, $S(V), \Lambda(V)$, etc., has on it a natural grading, and the degree on $\mathscr{A}(U, V)$ is formed by taking the sum of all these partial gradings. Thus if 
$\mathscr{A}(U, V)^{m}$ is the space of degree $m$ in $\mathscr{A}(U, V)$, we see $\mathscr{A}(U, V)^{m}$ is a direct sum of the spaces of the form

$$
S(V)^{\alpha_{1}} \otimes S(V)^{\alpha_{2}} \otimes \cdots \otimes S(V)^{\alpha_{k_{1}}} \otimes S\left(V^{*}\right)^{\beta_{1}} \otimes \cdots \otimes \Lambda\left(V^{*}\right)^{\delta_{l_{2}}},
$$

where $S(V)^{\alpha_{1}}$ is the space of degree $\alpha_{1}$ in $S(V)$, and so forth, and $\sum \alpha_{i}+$ $\sum \beta_{i}+\sum \gamma_{i}+\sum \delta_{i}=m$. The decomposition of $\mathscr{A}(U, V)$ into these subspaces is clearly a decomposition into submodules for $G$. Therefore the $G$-invariants will be the direct sum of the invariants contained in the spaces $(*)$. We therefore look at such a space.

A space of the form $(*)$ is a subspace of $\left(\otimes^{p} V\right) \otimes\left(\otimes^{q} V^{*}\right)$, where $p=$ $\sum \alpha_{i}+\sum \gamma_{i}$ and $q=\sum \beta_{i}+\sum \delta_{i}$. Hence we may apply Theorems $1 \mathrm{~A}$ and $1 \mathrm{~B}$. To ease the discussion, let us assume $G \simeq \mathrm{O}_{n}$ or $\mathrm{Sp}_{2 m}$. The case of $G \simeq \mathrm{Gl}_{n}$ is similar. Thus we take $\beta_{i}=\delta_{j}=0$. From Theorem 1B we know that a $G$-invariant on $\otimes^{p} V$ is a sum of those obtained by pairing the factors in some way and taking the tensor product of the degree 2 invariants in the various $(V \otimes V)$ 's thus formed. We are only interested in the restriction of these invariants to the subspace $(*)$. This means that, after selecting a pairing, we then symmetrize with respect to the appropriate blocks of $\alpha_{i}$ factors and antisymmetrize with respect to the various blocks of $\gamma_{j}$ factors. Since the symmetrization and antisymmetrization operations correspond to one-dimensional representations of the symmetric groups of the blocks, two pairings which are transformed into one another by the action of the symmetric groups of the blocks defining $(*)$ give the same restriction (up to sign) to $(*)$. Further, there are some taboos to observe with regard to matching within given blocks. That is, if $G \simeq \mathrm{O}_{n}$, then the invariant corresponding to a pairing is symmetric in each pair in the pairing so if both members of a given pair belong to a block which gets antisymmetrized, then the invariant dies on restriction to $(*)$. Upon inspecting the invariants that are left after taking these restrictions into account, we find that they are indeed accounted for by products of the invariants of degree two. This concludes our proof.

\section{QuANTUM ALGEBRA}

Take two vector spaces $U, W$, and form $\mathscr{A}(U, W)$. Since $\mathscr{A}(U, W)$ is an algebra it acts on itself by (left) multiplication, so there is a natural embedding of $\mathscr{A}$ in $\operatorname{End}(\mathscr{A})$. In particular, for every $x$ in $U$ or $W$ we have the multiplication operator

$$
M_{x}: y \rightarrow x y \text {. }
$$

There is also an action of $U^{*}$ and $W^{*}$ on $\mathscr{A}(U, V)$. This is not completely simple to describe and our treatment will be rather homely. Again see Tilgner [T] for greater elegance.

Consider $u^{*} \in U^{*}$ first. Write $\operatorname{ker} u^{*}=U_{0}$ and let $L$ be a line complementary to $U_{0}$ in $U$. We have an isomorphism $\mathscr{A}(U, W) \simeq S(L) \otimes \mathscr{A}\left(U_{0}, W\right)$. 
If $x \in L$ is nonzero, then $\mathscr{A}(U, W)$ is the span of elements $x^{n} y$ with $y \in \mathscr{A}\left(U_{0}, W\right)$. We define an operator $D_{u^{*}}$ on $\mathscr{A}(U, W)$ by the formula

$$
D_{u^{*}}\left(x^{n} y\right)=n u^{*}(x) x^{n-1} y .
$$

It is easy to check that $D_{u^{*}}$ is well defined and that it is a derivation, that is

$$
D_{u^{*}}(y z)=D_{u^{*}}(y) z+y D_{u^{*}}(z) \text { for any } y, z \in \mathscr{A}(U, W) \text {. }
$$

If $\mathscr{A}(U, W)$ is regarded as the $\Lambda(W)$-valued polynomials on $U^{*}$, then $D_{u^{*}}$ is just the directional derivative in the direction of $u^{*}$. A direct computation shows the $D_{u^{*}}$ commute among themselves. Moreover, if $[A B]=A B-B A$ is the commutator of $A$ and $B$, we have the commutator relation

$$
\left[D_{u^{*}}, M_{u}\right]=u^{*}(u) 1 \text { for } u^{*} \in U^{*} \text { and } u \in U \text {. }
$$

We may reformulate (3) as follows. Put $\tilde{U}=U \oplus U^{*}$ and let $\langle,\rangle^{\sim}$ be the antisymmetric extension to $\tilde{U}$ of the pairing between $U$ and $U^{*}$. Explicitly,

$$
\left\langle u_{1}+u_{1}^{*}, u_{2}+u_{2}^{*}\right\rangle^{\sim}=u_{1}^{*}\left(u_{2}\right)-u_{2}^{*}\left(u_{1}\right) .
$$

The direct sum of the maps $u \rightarrow M_{u}$ and $u^{*} \rightarrow D_{u^{*}}$ defines an embedding

$$
i: \tilde{U} \hookrightarrow \operatorname{End}(\mathscr{A}(U, W)) .
$$

With these conventions we have the formula

$$
[i(x), i(y)]=\langle x, y\rangle^{\sim} 1 \text { for } x, y \in \tilde{U} .
$$

Thus if we set $\mathscr{H}=i(\tilde{U}) \oplus \mathbf{C}$, where $\mathbf{C}$ is identified to multiples of 1 in $\operatorname{End}(\mathscr{A}(U, W))$, we see $\mathscr{H}$ is a two-step nilpotent Lie algebra, with $\mathbf{C}$ as center.

We can perform a similar construction using $W$, but the result is a Jordan algebra rather than a Lie algebra. If $w^{*} \in W^{*}$, we may construct $D_{w^{*}}$ in exactly the same manner as we used for $u^{*}$ in $U^{*}$, even to the extent of using formula (2), providing we remember $n$ is restricted to zero and 1 in this case. This endomorphism $D_{w^{*}}$ is usually called inner multiplication, at least in the case where $U=\{0\}$, so $\mathscr{A}$ reduces to an exterior algebra. It is not quite a derivation. Rather, it satisfies a law like

$$
\begin{aligned}
& D_{w^{*}}(x y z)=x\left(D_{w^{*}}(y) z+(-1)^{q} y D_{w^{*}}(z)\right. \\
& \quad \text { for } x \in S(U), y \in \Lambda^{q}(W), z \in \mathscr{A}(U, W) .
\end{aligned}
$$

Such a law is said to define an antiderivation or graded derivation. The law (7) makes it congenial to consider anticommutators rather than commutators. Define $\{A, B\}=A B+B A$. Then it is easy to check that there is an anticommutation relation

$$
\left\{D_{w^{*}}, M_{w}\right\}=w^{*}(w) 1 \text { for } w \in W \text { and } w^{*} \in W^{*} .
$$

Put $\tilde{W}=W \oplus W^{*}$ and let (, ) (note the round parentheses) be the symmetric extension to $\tilde{W}$ of the pairing between $W$ and $W^{*}$. That is,

$$
\left(w_{1}+w_{1}^{*}, w_{2}+w_{2}^{*}\right)^{\sim}=w_{1}^{*}\left(w_{2}\right)+w_{2}^{*}\left(w_{1}\right) .
$$


Again, let

$$
i: \tilde{W} \rightarrow \operatorname{End}(\mathscr{A}(U, W))
$$

be the direct sum of the $D$ and $M$ maps. Then, taking into account the anticommutativity of the $D_{w}$ *'s among themselves, and of the $M_{w}$ 's, we see

$$
\{i(x), i(y)\}=(x, y)^{\sim} 1 \text { for } x, y \in \tilde{W} .
$$

Thus if we put $\mathscr{T}=i(\tilde{W}) \oplus \mathbf{C}$, we see that $\mathscr{T}$ is a two-step nilpotent Jordan algebra, with $\mathbf{C}$ as center.

Thus, using the maps $M$ and $D$, we have built in $\operatorname{End}(\mathscr{A}(U, W))$ a Lie algebra $\mathscr{H}$ and Jordan algebra $\mathscr{T}$. It is easy to see that $\mathscr{H}$ and $\mathscr{T}$ commute with each other. Denote by $\operatorname{End}^{\circ}(\mathscr{A}(U, W))$ the subalgebra of $\operatorname{End}(\mathscr{A}(U, W))$ generated by $\mathscr{H}$ and $\mathscr{T} .^{2}$ Clearly $\mathscr{A}(U, W)$ is embedded in $\operatorname{End}^{\circ}(\mathscr{A}(U, W))$ via the left multiplication. Let $l: \mathscr{A}(U, W) \rightarrow \operatorname{End}^{\circ}(\mathscr{A}(U, W))$ denote this embedding. Also let $l^{*}: \mathscr{A}\left(U^{*}, W^{*}\right) \rightarrow \operatorname{End}^{\circ}(\mathscr{A}(U, W))$ be the homomorphism extending the above defined embedding of $U^{*} \oplus W^{*}$. The following is a straightforward inductive computation using formulas (4) and (8), or (6) and (11), above.

Theorem 3. The map $m: \mathscr{A}(U, V) \otimes \mathscr{A}\left(U^{*}, V^{*}\right) \rightarrow \operatorname{End}^{\circ}(\mathscr{A}(U, V))$ defined by $m(x \otimes y)=l(x) l^{*}(y)$ is a linear isomorphism. Further End $^{\circ}$ is isomorphic to the free algebra generated by $i(\tilde{U}) \oplus i(\tilde{W})$ subject to the relations (6) and (11) (plus commutativity between $i(\tilde{U})$ and $i(\tilde{W}))$.

Remark. From the universal description of End $^{\circ}$ given in Theorem 3, it is clear that $\operatorname{Sp}\left(\tilde{U},\langle,\rangle^{\sim}\right) \times \mathrm{O}\left(\tilde{W},(,)^{\sim}\right)$ acts as a group of automorphisms on End $^{\circ}$ and preserves the generating subspace $i(\tilde{U}) \oplus i(\tilde{W})$. It will be important for us to know that this action is essentially inner, and to compute it. These are our immediate goals.

We observe next that End $^{\circ}$ has a natural filtered structure. Indeed, we put End $^{\circ(0)}=\mathbf{C}$, and End ${ }^{\circ(1)}=\mathscr{H}+\mathscr{T}$, and inductively write

$$
\text { End }^{\circ(n+1)}=\operatorname{End}^{\circ(n)} \cdot \operatorname{End}^{\circ(1)}=\left\{\sum x y: x \in \operatorname{End}^{\circ(n)}, y \in \operatorname{End}^{\circ(1)}\right\} \text {. }
$$

Then in general End ${ }^{\circ(m)} \cdot$ End $^{\circ(n)}=$ End $^{\circ(n+m)}$. Consider End ${ }^{\circ(2)} /$ End $^{\circ(1)}$. This is isomorphic to $S^{2}(i(\tilde{U})) \oplus(\tilde{U} \otimes \tilde{W}) \oplus \Lambda^{2}(i(\tilde{W}))$.

Theorem 4. The subspace $S^{2}(i(\tilde{U})) \oplus \Lambda^{2}(i(\tilde{W}))=\mathfrak{g}$ of $\operatorname{End}^{\circ(2)} / \operatorname{End}^{\circ(1)}$ has a structure of Lie algebra. Precisely, the commutator relation $\left[\mathfrak{g}, \mathrm{End}^{\circ(n)}\right] \subseteq$ End $^{\circ(n)}$ holds. ${ }^{3}$ The Lie algebra structure is the obvious one arising from this

${ }^{2}$ A proper appelation for End $^{\circ}$ is problematical. Tilgner [T] calls it a graded Weyl algebra. It might as well be called a graded Clifford algebra. Or more symmetrically, Clifford-Weyl algebra or Weyl-Clifford algebra. These abbreviate to $\mathrm{CW}$ and WC respectively, each with its own drawbacks. Perhaps, after the manner of its generation, it could be termed the Ho-Jo algebra. The label I favor is quantum algebra. It has a certain aura, and it is consistent with other terminology inspired by physics. But I am not insisting yet.

${ }^{3}$ Here is one point where a thorough-going graded formulation would help a lot. We could assert $\left[\mathrm{End}^{\circ(m)}, \mathrm{End}^{\circ(n)}\right] \subseteq \mathrm{End}^{\circ(n+m-2)}$, where [ , ] would signify the graded commutator. 
(e.g., put $n=2$ ). Further the resulting action of $\mathfrak{g}$ on $\operatorname{End}^{\circ(1)} / \operatorname{End}^{\circ(0)} \simeq \tilde{U} \oplus \tilde{W}$ identifies $S^{2}(i(\tilde{U}))$ with the Lie algebra of the isometry group of $\langle,\rangle^{\sim}$, and identifies $\Lambda^{2}(i(\tilde{W}))$ with the Lie algebra of the isometry group of $(,)^{\sim}$.

Proof. All these facts are straightforward from formulas (4) and (8) or (6) and (11). In fact, if we verify the action on End $^{\circ(1)}$ is as it should be, the rest will follow. For example, take $a, b, c$ in $i(\tilde{W})$. Then $[a, b]=a b-b a \in \Lambda^{2}(i(\tilde{W}))$, and we compute

$$
\begin{aligned}
{[[a, b], c] } & =[b,[c, a]]+[a,[b, c]] \\
& =b c a-b a c-c a b+a c b+a b c-a c b-b c a+c b a \\
& =\{b, c\} a-b\{a, c\}-\{a, c\} b+a\{b, c\} \\
& =2 a(b, c)^{\sim}-2 b(a, c)^{\sim} .
\end{aligned}
$$

To check this transformation is an infinitesimal isometry of $(,)^{\sim}$ we compute

$$
\left(2 a(b, c)^{\sim}-2 b(a, c)^{\sim}, d\right)^{\sim}=2\left((a, d)^{\sim}(b, c)^{\sim}-(b, d)^{\sim}(a, c)^{\sim}\right) .
$$

The right-hand side of this is antisymmetric in $c$ and $d$, as it should be. The argument for $\tilde{U}$ is similar.

On the basis of the above theorem, we will denote $S^{2}(i(\tilde{U}))$ by $\mathfrak{s p}(\tilde{U})=\mathfrak{s p}$, and denote $\Lambda^{2}(i(\tilde{W}))$ by $\mathfrak{o}(\tilde{W})=\mathfrak{o}$. It is implicit in the computation given above, that if we take $s p$ to be the span of the anticommutators of elements of $i(\tilde{U})$, and take $\mathfrak{o}$ to be the span of commutators of elements of $i(\tilde{W})$, then

$$
[\mathfrak{g}, i(\tilde{U})+i(\tilde{W})]=i(\tilde{U})+i(\tilde{W}),
$$

where now $\mathfrak{g}=\mathfrak{s p} \oplus \mathfrak{o}$. Thus $\mathfrak{g}$ exists not merely inside End $^{\circ(2)} /$ End $^{\circ(1)}$ but as an actual Lie subalgebra of End $^{\circ}$. Because taking commutators with $\mathfrak{g}$ preserves the filtration on $\mathrm{End}^{\circ}$, we can exponentiate the resulting derivations. We state these facts formally.

Theorem 5. The Lie algebra $\mathfrak{g}=\mathfrak{s p} \oplus \mathfrak{o}$ may be embedded as a Lie subalgebra of End $^{\circ}$, and so acts as a Lie algebra of derivations of $\mathrm{End}^{\circ}$, preserving the subspace $i(\tilde{U}) \oplus i(\tilde{W})$ and the filtration $\left\{\mathrm{End}^{\circ(k)}\right\}$. Thus $\mathfrak{g}$ may be exponentiated to obtain an action by automorphisms of $\operatorname{Sp}\left(\tilde{U},\langle,\rangle^{\sim}\right) \times \mathrm{SO}\left(\tilde{W},(,)^{\sim}\right)$ on $\mathrm{End}^{\circ}$.

Now let us compute this action explicitly. From the filtration on $E^{\circ} d^{\circ}$ we may form the associated graded algebra

$$
\operatorname{GrEnd}^{\circ}=\bigoplus_{n=0}^{\infty} \operatorname{End}^{\mathrm{o}(n)} / \operatorname{End}^{\mathrm{o}(n-1)} .
$$

The multiplication on $\mathrm{GrEnd}^{\circ}$ is formed by taking the direct sum of the maps

$$
\bar{m}:\left(\operatorname{End}^{\circ(n)} / \operatorname{End}^{\circ(n-1)}\right) \times\left(\operatorname{End}^{\circ(m)} / \operatorname{End}^{\circ(m-1)}\right) \rightarrow \operatorname{End}^{\circ(n+m)} / \operatorname{End}^{\circ(n+m-1)} \text {, }
$$

where $\bar{m}$ is the map induced by multiplication in End $^{\circ}$. The next result is again an obvious consequence of formulas (4), (8), (6) and (11). 
Theorem 6. The map $i: U \oplus W \simeq$ End $^{\circ(1)} / \mathrm{End}^{\circ(0)}$ extends to an isomorphism of algebras

$$
\operatorname{Gr} \operatorname{End}^{\circ}(\mathscr{A}(U, W)) \simeq \mathscr{A}(\tilde{U}, \tilde{W}) .
$$

Moreover, the action of $\operatorname{Sp}(\tilde{U}) \times \mathrm{O}(\tilde{W})$ on $\mathrm{GrEnd}^{\circ}$ induced from the action by automorphisms on $\mathrm{End}^{\circ}$ is identified to the natural action of $\mathrm{Sp}(\tilde{U}) \times \mathrm{O}(\tilde{W})$ on $\mathscr{A}(\tilde{U}, \tilde{W})$.

Remark. It was convenient for our purposes to begin with $\mathscr{A}(U, W)$ and construct the endomorphism algebra $\operatorname{End}^{\circ}(\mathscr{A}(U, W))$. However, we could if we wished regard End ${ }^{\circ}$ as a primary object, an algebra attached to a pair $\tilde{U}, \tilde{W}$ of spaces, the first carrying a symplectic structure, the second an orthogonal structure. From such a point of view, how do we recapture $U$ and $W$ ? The answer is that $\mathscr{A}(U, W)$ provides an irreducible module for End ${ }^{\circ}$ of a particular type. Suppose $\left(U_{1}, U_{2}\right)$ is a pair of subspaces of $\tilde{U}$ such that $\tilde{U}=U_{1} \oplus U_{2}$ and each $U_{i}$ is isotropic for $\langle,\rangle^{\sim}$, i.e., the restriction of $\langle,\rangle^{\sim}$ to $U_{i}$ is identically zero. Let $\left(W_{1}, W_{2}\right)$ be a similar pair of subspaces of $\tilde{W}$. Then it is not hard to check that the subalgebra (with 1) $B_{i}$ of End ${ }^{\circ}$ generated by $U_{i} \oplus W_{i}$ is a copy of $\mathscr{A}\left(U_{i}, W_{i}\right)$ and that End ${ }^{\circ} \simeq B_{1} \otimes B_{2}$ via multiplication. Thus, if $L_{2}$ is the left ideal generated by $U_{2} \oplus W_{2}$, we see End $^{\circ} / L_{2} \simeq B_{1}$, so the natural left action of $\mathrm{End}^{\circ}$ on $\mathrm{End}^{\circ} / L_{2}$ can be interpreted as an action on $B_{1}$, with $B_{1}$ acting on itself by multiplication. Clearly the procedure we have gone through is precisely opposite to this.

Note further that by the above we have in particlar an action of $\mathfrak{g}=\mathfrak{s p} \oplus \mathfrak{o}$ on $B_{1}$. Since the action of $o$ takes place essentially on the finite-dimensional space $\mathscr{A}\left(W_{1}\right) \simeq \Lambda\left(W_{1}\right)$, this action will exponentiate to a group action. As is well known, the resulting group is not $\mathrm{SO}(\tilde{W})$, but its simply connected 2-fold cover $\operatorname{Spin}(\tilde{W})$. This is the standard construction of $\operatorname{Spin}(\tilde{W})$. The associated representation is called the Spin representation.

It turns out that the action of $\mathfrak{s p}$ will not exponentiate to a group action on $B_{1}$. However, the space $B_{1}$ may be embedded in a larger space, with a natural realization as a space of smooth (form-valued) functions (a seemingly intrinsically transcendental object), and the action of $\mathfrak{s p}$ may be suitably extended so that it does exponentiate to a group action. Over $\mathbf{C}$, where we are now, the resulting group is $\operatorname{Sp}(\tilde{U})$, as it must be since $\operatorname{Sp}(\tilde{U})$ is simply connected. However, a parallel construction may be carried out for any local field, and over all fields except $\mathbf{C}$, the result is a group which is a 2-fold cover of the usual symplectic group over the field. This construction was discovered by Segal, Shale and Weil [Sh, Wi2] and many terms are now used to describe it, e.g., Weil representation, Shale-Weil representation, metaplectic representation, harmonic. I would like to propose yet another term which seems to me more satisfactory than the above choices. I would refer to this representation as the oscillator representation, and the associated group as the oscillator group. Usually it is not necessary to justify terminology at length, it being arbitrary, etc., 
but because there is such a current welter of names, I note the following points in favor of this new one.

(a) The spin and oscillator representations are clearly intimately related, and in fact appear as two aspects of the same phenomenon.

(b) The term spin is colorful and is inspired by a physical phenomenon, namely the "spin" of the elementary particles, which the spin representation describes mathematically.

(c) The algebra associated with the oscillator representation also describes an important physical system, namely the (quantum mechanical) harmonic oscillator. (Indeed, the fact that the exponentiated representation (over $\mathbf{R}$ ) requres a 2-fold cover of $\mathrm{Sp}$ is physically interpreted as the existence of "zero-point energy", a basic phenomenon, and one that causes the first need to "renormalize" in quantum field theory.)

(d) The terms "spin" and "oscillator" are clearly consonant.

\section{Duality}

To return to business, let us recall that, with $\mathfrak{g} \simeq \mathfrak{s p} \oplus \mathfrak{o}$, we have

$$
\text { End }^{\circ(2)} / \text { End }^{\circ(1)} \simeq \mathfrak{g} \oplus(\tilde{U} \otimes \tilde{W})
$$

We know we can realize $\mathfrak{g}$ as an actual subspace of $\mathrm{End}^{\circ(2)}$. Then End $^{\circ(2)}$ is a module for $\mathfrak{g}$ and we can find a subspace isomorphic to $\tilde{U} \otimes \tilde{W}$. Thus the whole space $\mathfrak{g} \oplus(\tilde{U} \otimes \tilde{W})=\mathfrak{g r}$ may be regarded as a subspace of End $^{\circ}$, where $\mathfrak{g}$ is a Lie subalgebra under the induced bracket operation, and $\tilde{U} \otimes \tilde{W}$ is $\mathfrak{g}$-invariant. It turns out $\mathfrak{g r}$ has further structure. Namely, if you take the anticommutator of two elements of $\tilde{U} \otimes \tilde{W}$, the result belongs to $\mathfrak{g}$. This may again be easily checked using formulas (4), (8), (6), and (11). We cannot resist recording the relevant identity:

$$
\{a \otimes c, b \otimes d\}=\frac{1}{2}([a, b] \otimes[c, d]+\{a, b\} \otimes\{c, d\}) .
$$

The resulting structure on $\mathfrak{g r}$ is called a graded Lie algebra structure. (We note End $^{\circ(1)}$ is also a graded Lie algebra in a trivial sort of way.) Some physicists are quite interested in graded Lie algebras these days. Simple graded Lie algebras have been classified by $\mathrm{Kac}[\mathrm{K}]$ and $\mathfrak{g r}$ is on his list. Again, we note that this realization of $\mathfrak{g r}$ has also been noticed by Tilgner [T] and described more elegantly.

Write $\mathfrak{g r}_{0}=\mathfrak{g}$ and $\mathfrak{g r}_{1}=\tilde{U} \otimes \tilde{W}$. By a subalgebra of $\mathfrak{g r}$ we will mean a linear subspace $\Gamma$ of $\mathfrak{g r}$ such that

$$
\begin{gathered}
\Gamma=\left(\Gamma \cap \mathfrak{g r}_{0}\right) \oplus\left(\Gamma \cap \mathfrak{g r}_{1}\right)=\Gamma_{0} \oplus \Gamma_{1}, \\
{\left[\Gamma_{0}, \Gamma_{0}\right] \subseteq \Gamma_{0}, \quad\left[\Gamma_{0}, \Gamma_{1}\right] \subseteq \Gamma_{1}, \quad \text { and }\left\{\Gamma_{1}, \Gamma_{1}\right\} \subseteq \Gamma_{0} .}
\end{gathered}
$$

If $\Gamma$ and $\Gamma^{\prime}$ are two subalgebras, then we say $\Gamma$ and $\Gamma^{\prime}$ commute if all possible brackets with one element from $\Gamma$ and one from $\Gamma$ are zero. If $\Gamma$ is a subalgebra, let $\Gamma^{\prime}$ be the set of all elements commuting with $\Gamma$. We call $\Gamma^{\prime}$ the 
centralizer of $\Gamma$ in $\mathfrak{g r}$. It may be checked that $\Gamma^{\prime}$ is a subalgebra of $\mathfrak{g r}$. (Actually, there are some identities involved here, called graded Jacobi identities. These are

$$
\begin{aligned}
{[a,[b, c]] } & =[c,[b, a]]+[b,[a, c]], \\
{[a,\{b, c\}] } & =\{b,[a, c]\}+\{c,[a, b]\} \\
& =[\{a, c\}, b]+[\{b, a\}, c] .
\end{aligned}
$$

We already know that $\mathfrak{g r}_{0}=\mathfrak{g}$ acts on $i(\tilde{U}) \oplus i(\tilde{W})$ by the usual commutator action. We can also define an action of $\mathfrak{g r}_{1}$ on $i(\tilde{U}) \oplus i(\tilde{W})$ by defining

$$
\gamma(u \oplus w)=\{\gamma, w\} \oplus[\gamma, u] \text { for } \gamma \in \mathfrak{g r}_{1}, u \in i(\tilde{U}), w \in i(\tilde{W}) .
$$

It may be checked, again using the graded Jacobi identities, that the actions of $\mathfrak{g r}_{0}$ and $\mathfrak{g r}_{1}$ fit together to form a representation of $\mathfrak{g r}$, in the sense that all the relevant commutator and anticommutator relations are satisfied. If $\Gamma \subseteq \mathfrak{g r}$ is a subalgebra, we will say $\Gamma$ is reductive if $\Gamma$ acts reductively on $i(\tilde{U}) \oplus i(\tilde{W})$.

Let $\left(\Gamma, \Gamma^{\prime}\right)$ be a pair of subalgebras. We say $\Gamma$ and $\Gamma^{\prime}$ are in duality or form a dual pair if $\Gamma^{\prime}$ is the centralizer of $\Gamma$ and vice versa. We say $\left(\Gamma, \Gamma^{\prime}\right)$ is a reductive dual pair if $\left(\Gamma, \Gamma^{\prime}\right)$ is a dual pair and each of $\Gamma, \Gamma^{\prime}$ is reductive.

We will give three examples of reductive dual pairs, corresponding to the three classical groups. It seems most likely that these are essentially the only examples of reductive dual pairs in $\mathfrak{g r}$, but this result is not crucial to our development and would take us too far afield into graded Lie algebras. (It is fairly easy to show these exhaust irreducible pairs $\left(\Gamma, \Gamma^{\prime}\right)$ such that $\Gamma \subseteq \mathfrak{g r}_{0}$.)

First, observe that $\mathrm{GL}(U) \times \mathrm{GL}(W)=\mathrm{GL}$ acts on $\operatorname{End}^{\circ}(\mathscr{A}(U, W))$ by automorphisms. For GL acts on $\mathscr{A}(U, W)$ by automorphisms, and if $x \in$ $\operatorname{End}^{\circ}(\mathscr{A}(U, W))$ and $g \in \mathrm{GL}$, then $g x g^{-1}$, the conjugate of $x$ by $g$, is again in End $^{\circ}$. Indeed, it is clear that conjugation by $g$ preserves the space $i(\tilde{U}) \times i(\tilde{W})$. More particularly, one has relations like

$$
g M_{x} g^{-1}=M_{g(u)} g D_{y} g^{-1}=D_{g(y)}
$$

for $x \in U \oplus W$ and $y \in U^{*} \oplus W^{*}$, where $g(x)$ and $g(y)$ are the standard actions of $g \in \mathrm{GL}$ on $U \oplus W$ and $U^{*} \oplus W^{*}$. Thus we have an embedding GL $\rightarrow \mathrm{Sp} \times 0$ given by conjugation as above. There is a corresponding infinitesimal embedding $\mathfrak{g l}(U) \times \mathfrak{g l}(W) \rightarrow \mathfrak{s p} \times \mathfrak{o}=\mathfrak{g r}_{0}$. In fact, this embedding already arises from an embedding of $\mathfrak{g l}=\mathfrak{g l}(U) \times \mathfrak{g l}(W)$ in $\operatorname{End}^{\circ(2)}$. Indeed, if we differentiate the action of GL on $\mathscr{A}(U, V)$, the endomorphisms of $\mathscr{A}(U, V)$ describing the resulting action of $\mathfrak{g l}$ are easily seen to belong to End $^{\circ(2)}$, and conjugation by GL on End ${ }^{\circ}$ is the inner action arising from exponentiating the bracket action by this image of $\mathfrak{g l}$. As a complement, note that $\mathfrak{s p} \simeq S^{2}(\tilde{U}) \simeq S^{2}(U) \oplus\left(U \otimes U^{*}\right) \oplus S^{2}\left(U^{*}\right)$. Of course $U \otimes U^{*} \simeq \mathfrak{g l}(U)$ as Lie algebras, and indeed $U \otimes U^{*}$ is precisely the image of the above embedding of $\mathfrak{g l}(U)$.

Now consider a classical group $G$ with standard module $V$. Put $U=$ $\left(\oplus^{k_{1}} V\right) \oplus\left(\oplus^{l_{1}} V^{*}\right)=V^{k_{1}} \oplus V^{* l_{1}}$, and put $W=V^{k_{2}} \oplus V^{* l_{2}}$. Let $G$ act on 
$U \oplus W$ by the direct sum action. This gives an embedding $G \rightarrow$ GL. If $\Gamma$ is the Lie algebra of $G$, then according to the preceding discussion, we have a corresponding inclusion $\Gamma \hookrightarrow \mathfrak{g r}_{0}$. Let $\Gamma^{\prime}$ be the centralizer of $\Gamma$ in $\mathfrak{g r}$. Then we claim $\left(\Gamma, \Gamma^{\prime}\right)$ forms a reductive dual pair. We will show this by giving a more complete discussion which will yield an explicit description of $\Gamma^{\prime}$.

We consider the groups one at a time. First take $G=\mathrm{GL}(V)$. Then we may write $U \simeq\left(V \otimes D_{1}\right) \oplus\left(V^{*} \otimes E_{1}\right)$, where $D_{1}$ and $E_{1}$ are auxiliary spaces of the appropriate dimensions. Similarly, we have $W \simeq\left(V \otimes D_{2}\right) \oplus\left(V^{*} \otimes E_{2}\right)$. Then

$$
\begin{aligned}
\tilde{U} & =U \oplus U^{*}=\left(V \otimes D_{1}\right) \oplus\left(V^{*} \otimes E_{1}\right) \oplus\left(V^{*} \otimes D_{1}^{*}\right) \oplus\left(V \otimes E_{1}^{*}\right) \\
& =V \otimes\left(D_{1} \oplus E_{1}^{*}\right) \oplus V^{*} \otimes\left(D_{1} \oplus E_{1}^{*}\right)^{*}=U^{\prime} \oplus U^{\prime *} .
\end{aligned}
$$

It is immediate from the definition of the form $\langle,\rangle^{\sim}$ that $\mathrm{GL}\left(D_{1} \oplus E_{1}^{*}\right)$ is inside $\operatorname{Sp}(\tilde{U})$. In fact, if $x \in \mathrm{GL}(\tilde{U})$ commutes with $\mathrm{GL}(V)$, it preserves $U^{\prime}$ and $U^{\prime *}$, and $x_{\mid U^{\prime}}$ must belong to $\mathrm{GL}\left(D_{1} \oplus E_{1}^{*}\right)$. For $x$ to preserve $\langle,\rangle^{\sim}$, we must also require that $x_{\mid U^{\prime *}}$ be the inverse transpose of $x_{\mid U^{\prime}}$. Thus $\operatorname{GL}\left(D_{1} \oplus E_{1}^{*}\right)$ is the full centralizer of $\mathrm{GL}(V)$ in $\operatorname{Sp}(\tilde{U})$. Since the situation is symmetric in $V$ and $D_{1} \oplus E^{*}$, we see that $\mathrm{GL}(V)$ is the centralizer of $\mathrm{GL}\left(D_{1} \oplus E_{1}^{*}\right)$ in $\operatorname{Sp}(\tilde{U})$. Thus we could say that $\operatorname{GL}(V)$ and $\operatorname{GL}\left(D_{1} \oplus E_{1}^{*}\right)$ form a reductive dual pair in $\operatorname{Sp}(\tilde{U})$. Indeed, if $W=\{0\}$, then $\mathfrak{g r}$ reduces to $\mathfrak{s p}(\tilde{U})$ and we are just discussing the infinitesimal version of this pair. That is, $\mathfrak{g l}(V)$ and $\mathfrak{g l}\left(D_{1} \oplus E_{1}^{*}\right)$ are mutual centralizers in $\mathfrak{s p}$.

Next considering $W \simeq\left(V \otimes D_{2}\right) \oplus\left(V^{*} \otimes E_{2}\right)$, we find in a similar manner that $\mathfrak{g l}(V)$ and $\mathfrak{g l}\left(D_{2} \oplus E_{2}^{*}\right)$ are in duality in $\mathfrak{o}=\mathfrak{o}(W)$. Thus if $\Gamma=\mathfrak{g l}(V)$, we have $\Gamma^{\prime}=\Gamma_{0}^{\prime} \oplus \Gamma_{1}^{\prime}$ and we have determined that $\Gamma_{0}^{\prime} \simeq \mathfrak{g l}\left(D_{1} \oplus E_{1}^{*}\right) \oplus$ $\mathfrak{g l}\left(D_{2} \oplus E_{2}^{*}\right)$.

We turn to the computation of $\Gamma_{1}^{\prime}$. We have $\mathfrak{g}_{1}=\tilde{U} \otimes \tilde{W} \simeq \operatorname{Hom}(\tilde{U}, \tilde{W}) \simeq$ $\operatorname{Hom}(\tilde{W}, \tilde{U})$. The action of $\mathrm{Sp} \times \mathrm{O}$ on $\mathfrak{g r}_{1}$ is the tensor product of the standard actions, and the bracket operation of $\mathfrak{g r}_{0} \simeq \mathfrak{s p} \oplus \mathfrak{o}$ on $\mathfrak{g r}_{1}$ is just the infinitesimal version of this action. Thus if we write $\mathfrak{g r}_{1}$ as $\operatorname{Hom}(\tilde{U}, \tilde{W})$, then clearly $\Gamma_{1}^{\prime}$ may be thought of as the $\mathrm{GL}(V)$-intertwining operators between $\tilde{U}$ and $\tilde{V}$. In terms of the above discussion, this would be

$$
\Gamma_{1}^{\prime} \simeq \operatorname{Hom}\left(D_{1} \oplus E_{1}^{*}, D_{2} \oplus E_{2}^{*}\right) \oplus \operatorname{Hom}\left(D_{1}^{*} \oplus E_{1}, D_{2}^{*} \oplus E_{2}\right) .
$$

Finally, we can see that $\Gamma=\mathfrak{g} 1(V)$ is the full centralizer of $\Gamma^{\prime}$. It is evident that the centralizer of $\Gamma_{0}^{\prime}$ in $\mathfrak{s p} \oplus \mathfrak{o}$ is $\mathfrak{g} 1(V) \oplus \mathfrak{g} 1(V)$. Inside this algebra only the diagonal can also centralize any nonzero element of $\Gamma_{1}^{\prime}$. Thus $\Gamma=\mathfrak{g} 1(V)=$ $\left(\Gamma^{\prime}\right)_{0}^{\prime}$. Since $\Gamma_{1}^{\prime}$ is the direct sum of two algebras, one acting trivially on $\tilde{W}$ and without fixed points on $\tilde{U}$, and vice versa for the other, we see $\left(\Gamma^{\prime}\right)_{1}^{\prime}=\{0\}$. Thus indeed $\Gamma$ is the centralizer of $\Gamma^{\prime}$, and $\left(\Gamma, \Gamma^{\prime}\right)$ are a reductive dual pair in $\mathfrak{g r}$.

We now consider $G=\operatorname{Sp}(V)$. Then again we may write $U=V \otimes D_{1}$ where $D_{1}$ is an auxiliary space. (We can leave $V^{*}$ out of it.) Then

$$
\tilde{U}=U \oplus U^{*}=\left(V \otimes D_{1}\right) \oplus\left(V^{*} \otimes D_{1}^{*}\right) \simeq V \otimes\left(D_{1} \oplus D_{1}^{*}\right) .
$$


Furthermore, the symplectic form $\langle,\rangle^{\sim}$ on $\tilde{U}$ can be written as the tensor product of $\langle$,$\rangle , the given form on V$, with the natural symmetric form $(,)_{1}$ on $D_{1} \otimes D_{1}^{*}$. From this factorization of $\tilde{U}$ it is easy to see that the centralizer of $\operatorname{Sp}(V)$ in $\operatorname{Sp}(\tilde{U})$ is $\mathrm{O}\left(D_{1} \oplus D_{1}^{*},(,)_{1}\right)$ and that $\operatorname{Sp}(V)$ and $\mathrm{O}\left(D_{1} \oplus D_{1}^{*}\right)$ form a reductive dual pair in $\operatorname{Sp}(\tilde{U})$. This construction may be refined somewhat. Instead of taking $U=V \otimes D_{1}$, we may write directly $\tilde{U} \simeq V \otimes D_{1}^{\prime}$, where $D_{1}^{\prime}$ is a space with a symmetric bilinear form on it. Here too it is easy to see that $\operatorname{Sp}(V)$ and $\mathrm{O}\left(D_{1}^{\prime}\right)$ are mutual centralizers in $\operatorname{Sp}(\tilde{U})$. This procedure is equivalent to the old one if $D_{1}^{\prime}$ is isomorphic to $D_{1} \oplus D_{1}^{*}$ for some $D_{1}$. Since we are working over $\mathbf{C}$, this will be the case if $\operatorname{dim} D_{1}^{\prime}$ is even, but if $\operatorname{dim} D_{1}^{\prime}$ is odd, it will not be so.

Next write $W=V \otimes D_{2}$, so that $\tilde{W} \simeq V \otimes\left(D_{2} \oplus D_{2}^{*}\right)$. If we put on $D_{2} \oplus D_{2}^{*}$ the natural symplectic structure $\langle,\rangle_{2}$, then we have $(,)^{\sim}=\langle,\rangle \otimes\langle,\rangle_{2}$. Thus now we see $\operatorname{Sp}(V)$ and $\operatorname{Sp}\left(D_{2} \oplus D_{2}^{\prime}\right)$ are a reductive dual pair in $\mathrm{O}(\tilde{W})$.

Taking the infinitesimal analogs, we find that if $\Gamma \subseteq \mathfrak{g r}_{0}$ is the Lie algebra of $\mathrm{Sp}(V)$ embedded in the manner indicated above, then

$$
\Gamma_{0}^{\prime} \simeq \mathfrak{o}\left(D_{1}^{\prime}\right) \oplus \mathfrak{s p}\left(D_{2} \oplus D_{2}^{*}\right)
$$

Again $\Gamma_{1}^{\prime}$ is the space of $\operatorname{Sp}(V)$ intertwining maps from $\tilde{U}$ to $\tilde{W}$, and is isomorphic to $D_{1}^{\prime} \otimes\left(D_{2} \oplus D_{2}^{*}\right)$; and again $\left(\Gamma, \Gamma^{\prime}\right)$ form a reductive dual pair.

Finally, we sketch the situation for an orthogonal group. Again write $U \simeq$ $V \otimes D_{1}$ and $W \simeq V \otimes D_{2}$; then $\tilde{U} \simeq V \otimes\left(D_{1} \oplus D_{1}^{*}\right)$ and $\tilde{W} \simeq V \otimes\left(D_{2} \oplus D_{2}^{*}\right)$. Now $\langle,\rangle^{\sim}$ on $\tilde{U}$ may be regarded as the tensor product of the symmetric form $($,$) on V$ and the natural antisymmetric form on $D_{1} \oplus D_{1}^{*}$, and $\mathrm{O}(V)$ and $\operatorname{Sp}\left(D_{1} \oplus D_{1}^{*}\right)$ form a dual pair in $\operatorname{Sp}(\tilde{U})$. Similarly, $(, ;$ on $\tilde{W}$ is the tensor product of $($,$) on V$ and the natural symmetric form on $D_{2} \oplus D_{2}^{*}$, and then $\mathrm{O}(V)$ and $\mathrm{O}\left(D_{2} \oplus D_{2}^{*}\right)$ are in duality in $\mathrm{O}(\tilde{W})$. Again we may note a refinement. If $\operatorname{dim} V$ is even, then $\mathrm{O}\left(D_{2} \oplus D_{2}^{*}\right)$ may be replaced by any $\mathrm{O}\left(D_{2}^{\prime}\right)$, where $D_{2}^{\prime}$ is a space with symmetric form, of odd or even dimension. Then if $\mathfrak{o}(V) \simeq \Gamma \subseteq \mathfrak{g r}_{0}$, we see $\Gamma_{0}^{\prime} \simeq \mathfrak{s p}\left(D_{1} \oplus D_{1}^{*}\right) \oplus \mathfrak{o}\left(D_{2}^{\prime}\right)$. Again $\Gamma^{\prime}$ is the space of $\mathrm{O}(V)$ operators from $\tilde{U}$ to $\tilde{W}$, essentially isomorphic to $\left(D_{1} \oplus D_{1}^{*}\right) \otimes D_{2}^{\prime}$. Again $\Gamma$ and $\Gamma^{\prime}$ are in duality.

In sum then, we have constructed a set of reductive dual pairs $\left(\Gamma, \Gamma^{\prime}\right)$ attached to classical groups. We will refer to them as the classical dual pairs. Note that in the general case, with neither $U$ nor $W$ trivial, there is a certain amount of asymmetry in the pairs as constructed. Indeed, in all the pairs $\Gamma \subseteq \mathfrak{g r}_{0}$, and $\Gamma_{0}=\Gamma$ is a simple Lie algebra (if we ignore the center of GL) while $\Gamma_{1}^{\prime}$ is nontrivial, and $\Gamma_{0}^{\prime}$ is the sum of two simple factors. However, if $U$ or $W$ is trivial, then the relation between $\Gamma$ and $\Gamma^{\prime}$ is essentially symmetric.

Having constructed the classical reductive dual pairs, we should now state a theorem about them. 
Theorem 7. Let $\left(\Gamma, \Gamma^{\prime}\right)$ be a classical reductive dual pair, with $\Gamma \subseteq \mathfrak{g r}_{0} \simeq \mathfrak{s p} \oplus \mathfrak{o}$. Let $G \subseteq \mathrm{Sp} \times \mathrm{O}$ be the classical group corresponding to $\Gamma$. (That is, $G$ is the subgroup of $\mathrm{Sp} \times \mathrm{O}$ leaving $\Gamma^{\prime}$ pointwise fixed.) Then the associative subalgebra of End ${ }^{\circ}$ generated by $\Gamma^{\prime}$ is the full algebra of $G$ invariants in End $^{\circ}$.

Proof. Consider the isomorphism $\mathrm{GrEnd}^{\circ} \simeq \mathscr{A}(\tilde{U}, \tilde{W})$ given by Theorem 6. This isomorphism is $(\mathrm{Sp} \times \mathrm{O})$-equivariant. From the description of reductive dual pairs given above, it is evident that the action of $G$ induced on $\mathrm{Gr} \mathrm{End}^{\circ}$, when transferred to $\mathfrak{a}(\tilde{U}, \tilde{W})$, becomes the action of $G$ attached to the direct sum of copies of the standard module for $G$ or its contragredient. Thus by Theorem 2, the $G$-invariants in $\mathrm{GrEnd}^{\circ}$ are generated by the degree 2 invariants. But under the identification $\mathfrak{g r} \simeq \mathrm{End}^{\circ(2)} / \mathrm{End}^{\circ(1)}$, the degree 2 invariants are just $\Gamma^{\prime}$. Now the result follows by a straightforward induction. Suppose $\Gamma^{\prime}$ generates all the $G$-invariants inside End $^{\circ(k)}$. Take a $G$-invariant $x$ in $\operatorname{End}^{\mathrm{o}(k+1)}$; then the image $\bar{x}$ of $x$ in $\operatorname{End}^{\mathrm{o}(k+1)} / \operatorname{End}^{\mathrm{o}(k)}$ can be written

$$
\bar{x}=\sum \bar{y}_{i} \bar{z}_{i},
$$

where the $y_{i}$ are in $\Gamma^{\prime}$ and the $\bar{z}_{i}$ are $G$-invariants in $\operatorname{End}^{\circ(k-1)} / \mathrm{End}^{\mathrm{o}(k-2)}$. We can lift the $\bar{z}_{i}$ to $G$-invariants $z_{i}$ in End ${ }^{\mathrm{o}(k-1)}$. Then clearly $x-\sum y_{i} z_{i}$ belongs to $E n d^{o(k)}$ and so by induction belongs to the algebra generated by $\Gamma^{\prime}$, as do the $z_{i}$. Hence so does $x$, and we are done.

If $G$ is connected (i.e., if $G$ is not an orthogonal group), then the $G$ invariants in $E \mathrm{Ed}^{\circ}$ are the same as the algebra commuting with $\Gamma$. In any case, the algebras generated by $\Gamma$ and $\Gamma^{\prime}$ commute with each other, and are close to being mutual commutants. In such a situation, one expects a dual relationship between the actions of the two algebras-a matching up of isotypic components, joint irreducible actions, and so forth. This is indeed true here.

Recall that for a given representation of a group on a vector space, an isotypic component is the sum of all subrepresentations isomorphic with some given irreducible representation of the group.

Theorem 8. Let $G$ be a classical group with standard module $V$. Let $U$ and $W$ be direct sums of copies of $V$ and $V^{*}$, and consider the resulting action of $G$ on $\mathscr{A}(U, W)$. Let

$$
\mathscr{A}(U, W)=\bigoplus_{j=0}^{\infty} I_{j}
$$

be the decomposition of $\mathscr{A}(U, W)$ into isotypic components for $G$. Let $\Gamma \subseteq \mathfrak{g r}$ be the image of the Lie algebra of $G$ corresponding to this action, and let $\Gamma^{\prime}$ be the centralizer of $\Gamma$ in $\mathfrak{g r}$. Then the joint action of $G$ and $\Gamma$ on any $I_{j}$ is irreducible, and $I_{j}$ has the form

$$
I_{j} \simeq \sigma_{j} \otimes \tau_{j},
$$


where $\sigma_{j}$ is a finite-dimensional representation of $G$ and $\tau_{j}$ is an irreducible module for $\Gamma^{\prime}$. Moreover, $\sigma_{j}$ and $\tau_{j}$ determine each other, so the correspondence $\sigma_{j} \leftrightarrow \tau_{j}$ is bijective.

Proof. If $\operatorname{End}^{\circ}(\mathscr{A}(U, W))$ were the full endomorphism ring of $\mathscr{A}(U, W)$, this would be immediate from Theorem 7 . Instead we need the following easy technical lemmas.

Lemma 1. If $X \subseteq \mathscr{A}(U, W)$ is a finite-dimensional subspace, then the restriction map $r: \operatorname{End}^{\circ}(\mathscr{A}(U, W) \rightarrow \operatorname{Hom}(X, \mathscr{A}(U, W))$ is surjective.

Lemma 2. Let $\mathscr{I}(G) \subseteq \operatorname{End}^{\circ}(\mathscr{A}(U, W))$ be the operators commuting with $G$. Then there is a linear projection operator $T \rightarrow T^{\#}$ of $\mathrm{End}^{\circ}$ onto $\mathscr{I}(G)$ with the following properties:

(i) $\left(S T^{\#}\right)^{\#}=S^{\#} T^{\#}$.

(ii) if $X \subseteq \mathscr{A}(U, W)$ is a G-invariant subspace and $T \in \mathrm{End}^{\circ}$ leaves $X$ invariant and commutes with $G$ on $X$, then $T_{\mid X}^{\#}=T_{\mid X}$.

The proof of Lemma 1 is straightforward. The proof of Lemma 2 is by the well-known "unitarian trick" of averaging over the maximal compact subgroup of $G$.

The theorem now follows directly from these facts. For let $X \subseteq I_{j}$ be a finite-dimensional $G$-invariant subspace. Then the joint action of $G$ and the commutant of $G$ in $\operatorname{End}(X)$ is irreducible on $X$ by the usual double commutant theorem. But the above lemmas imply that $\mathscr{I}(G)$ restricted to $X$ includes the commutant of $G$ in $\operatorname{End}(X)$. Since $X$ was arbitrarily large, this means the joint action of $\mathscr{I}(G)$ and $G$ on $I_{j}$ is irreducible. Similarly, if $I_{j} \neq I_{k}$ and $X \subseteq I_{j}$ and $Y \subseteq I_{k}$ are finite dimensional and $G$-invariant, then again by the lemmas there is an element of $\mathscr{I}(G)$ which is the identity on $X$ and zero on $Y$. Hence the representations of $\mathscr{I}(G)$, and thus of $\Gamma^{\prime}$ on $I_{j}$ and $I_{k}$, are distinct. This concludes the proof of the theorem.

It turns out that the $\Gamma^{\prime}$-modules occurring in $\mathscr{A}(U, W)$ have a characteristic structure which we will now describe. Recall the isomorphisms $\mathfrak{g r}_{0} \simeq S^{2}(\widetilde{U}) \oplus$ $\Lambda^{2}(\widetilde{W})$ and $\mathfrak{g r}_{1} \simeq \widetilde{U} \otimes \widetilde{W}$. Furthermore, $\widetilde{U} \simeq U \oplus U^{*}$ and $\widetilde{W} \simeq W \oplus W^{*}$, so that

$$
\mathfrak{g r}_{0} \simeq\left\{S^{2}(U) \oplus\left(U \otimes U^{*}\right) \oplus S^{2}\left(U^{*}\right)\right\} \oplus\left\{\Lambda^{2}(W) \oplus\left(W \otimes W^{*}\right) \oplus \Lambda^{2}\left(W^{*}\right)\right\}
$$

and $\mathfrak{g r}_{1} \simeq(U \otimes W) \oplus\left(U \otimes W^{*} \oplus U \otimes W\right) \oplus\left(U^{*} \otimes W^{*}\right)$. We define

$$
\begin{aligned}
& \mathfrak{g r}^{(2,0)} \simeq S^{2}(U) \oplus \Lambda^{2}(U) \oplus(U \otimes W), \\
& \mathfrak{g r}^{(1,1)} \simeq\left(U \otimes U^{*}\right) \oplus\left(W \otimes W^{*}\right) \oplus\left(U \otimes W^{*} \oplus U^{*} \otimes W\right), \\
& \mathfrak{g r}^{(0,2)} \simeq S^{2}\left(U^{*}\right) \oplus \Lambda^{2}\left(U^{*}\right) \oplus\left(U^{*} \otimes W^{*}\right) .
\end{aligned}
$$

It may be checked that $\mathfrak{g r}^{(2,0)}$ etc. are subalgebras of $\mathfrak{g r}$. Moreover $\mathfrak{g r}^{(2,0)}$ and $\mathfrak{g r}^{(0,2)}$ are abelian, in the sense that all brackets are zero. Also $\mathfrak{g r}^{(0,2)}$ and $\mathfrak{g r}^{(2,0)}$ 
are normalized by $\mathfrak{g r}^{(1,1)}$ in the sense that they are invariant under bracketing with $\mathfrak{g r}^{(1,1)}$. In terms of the action on $\mathfrak{g r}$ on $\mathscr{A}(U, W)$, the elements of $\mathfrak{g r}^{(2,0)}$ raise degree in $\mathscr{A}(U, W)$ by 2 . Elements of $\mathfrak{g}^{(0,2)}$ lower degree by 2 , and elements of $\mathfrak{g}^{(1,1)}$ preserve degree.

If $\left(\Gamma, \Gamma^{\prime}\right)$ is a classical reductive dual pair in $\mathfrak{g r}$ such that $\Gamma$ arises from a sum of actions on $U \oplus W$, then $\Gamma^{\prime}=\Gamma^{(2,0)} \oplus \Gamma^{(1,1)} \oplus \Gamma^{(0,2)}$, where $\Gamma^{(2,0)}=$ $\Gamma^{\prime} \cap \operatorname{gr}^{(2,0)}$, etc. Further, observe that $\Gamma^{(2,0)}$ is essentially the space of degree 2 invariants for $G$ in $\mathscr{A}(U, W)$, whereas $\Gamma^{(0,2)}$ might be thought of as the $G$-invariant "constant coefficient differential operators" of degree 2. (This is true in the traditional sense if $W=\{0\}$.) In any case, we will define

$$
\mathscr{H}=\left\{x \in \mathscr{A}(U, W) ; T(x)=0 \text { for all } T \in \Gamma^{(0,2)}\right\} .
$$

We will refer to $\mathscr{H}$ as the harmonics, or the $\Gamma^{\prime}$-harmonics.

Theorem 9. Let $G$ act on $\mathscr{A}(U, W)$ as in Theorem 8. Let

$$
\mathscr{A}(U, W) \simeq \bigoplus_{j=0}^{\infty} I_{j} \simeq \bigoplus_{j=0}^{\infty} \sigma_{j} \otimes \tau_{j}
$$

be the decomposition of $\mathscr{A}(U, W)$ into irreducible joint $\left(G, \Gamma^{\prime}\right)$-modules. Let $I_{0}$ be the G-invariants, and $\mathscr{H}$ the $\Gamma^{\prime}$-harmonics. Then

(i) The map $I_{0} \otimes \mathscr{H} \rightarrow \mathscr{A}(U, W)$ given by multiplication is surjective.

(ii) If $\mathscr{H}_{j}=\mathscr{H} \cap I_{j}$, then $\mathscr{H}=\bigoplus_{j=0}^{\infty} \mathscr{H}_{j}$ and $\mathscr{H}_{j} \neq\{0\}$ for any $j$. Also $I_{j}=I_{0} \mathscr{H}_{j} . \mathscr{H}_{j}$ consists of the elements in $I_{j}$ of lowest degree.

(iii) Each $\mathscr{H}_{j}$ is invariant under $\Gamma^{(1,1)}$, and forms an irreducible module for the joint action of $G$ and $\Gamma^{(1,1)}$. The resulting correspondence between G-modules and $\Gamma^{(1,1)}$-modules is again bijective. Furthermore, $\mathscr{H}_{j}$ forms a cyclic subspace for $I_{j}$ under the action of $\Gamma^{(2,0)}$. (Thus these are modules for $\Gamma^{\prime}$ with "highest weights".)

Proof. Since every element of $\mathscr{A}(U, W)$ is eventually killed by successive applications of elements from $\Gamma^{(0,2)}$, and since each $I_{j}$ is $\Gamma^{(0,2)}$-invariant, we see $\mathscr{H}_{j} \neq\{0\}$. Indeed, the elements of $I_{j}$ of lowest possible degree must be in $\mathscr{H}_{j}$. Also, since $\Gamma^{(0,2)}$ commutes with $G$, we know $\mathscr{H}$ will be $G$-invariant. This proves the first statement of (ii).

Let $\mathscr{I}^{(2,0)}, \mathscr{I}^{(1,1)}$ and $\mathscr{I}^{(0,2)}$ be algebras in $\operatorname{End}^{\circ}(\mathscr{A}(U, W))$ generated by $\Gamma^{(2,0)}, \Gamma^{(1,1)}$ and $\Gamma^{(0,2)}$ respectively. By virtue of the bracket relations in $\mathfrak{g r}$, we may write

$$
\mathscr{I}(G) \simeq \mathscr{I}^{(2,0)} \mathscr{I}^{(1,1)} \mathscr{I}^{(0,2)} .
$$

(This is the easy half of PBW.) Since $\Gamma^{(1,1)}$ normalizes $\Gamma^{(0,2)}$ it is clear that $\mathscr{H}_{j}$ is invariant under $\Gamma^{(1,1)}$, hence under $\mathscr{I}^{(1,1)}$. Thus the above decomposition of $\mathscr{F}(G)$ implies $I_{j}=\mathscr{I}^{(2,0)} \mathscr{H}_{j}$. This proves the third statement of (iii). Since 
as we have noted $\mathscr{J}^{(2,0)} \simeq I_{0}$, this also proves (i) and the second statement of (ii). Now suppose $X \subseteq \mathscr{H}_{j}$ is a homogeneous subspace invariant under $G$ and under $\Gamma^{(1,1)}$. Then by the irreducibility of the joint $\left(G, \Gamma^{\prime}\right)$-action, we have $I_{j}=\mathscr{I}(G) \cdot X=\mathscr{I}^{(2,0)} \cdot X$. But $\mathscr{I}^{(2,0)} \cdot X=X \oplus Y$, where $Y$ consists of elements of degree greater than the degree of $X$, since multiplication by elements of $\Gamma^{(2,0)}$ raises degree. Thus $X$ must consist of all elements of $I_{j}$ of minimum degree. The rest of the theorem follows, except for bijectivity of $G$-modules and $\Gamma^{(1,1)}$-modules defined by the $\mathscr{H}_{m}$. But it is easy to see that $\Gamma^{(1,1)}$ generates the subalgebra of $\operatorname{End}(\mathscr{H})$ commuting with $G$ and coming from End $^{\circ}$. So we are done.

\section{EXAMPLES}

(a) Classical theory of spherical harmonics. Let $G=\mathrm{O}_{n}(\mathbf{C})$ be the isometries of the inner product $\sum_{i=0}^{n} z_{i}^{2}$ on $\mathbf{C}^{n}$. This may be regarded as the complexification of the action of the compact group $\mathrm{O}_{n}(\mathbf{R})$ on $\mathbf{R}^{n}$. Consider the induced action on the polynomials on $\mathbf{C}^{n}$. This is just $\mathscr{A}(U, W)$ when $U=\mathbf{C}^{n}$ and $W=$ $\{0\}$. The function $r^{2}=\sum_{i=0}^{n} z_{i}^{2}$ is by definition an invariant polynomial. According to Theorem 2, it generates the algebra of $\mathrm{O}_{n}$ invariants. Thus

$$
I_{0}\left(\mathrm{O}_{n}\right) \simeq\left\{p\left(r^{2}\right), p \text { a polynomial }\right\} \text {. }
$$

The Laplacian $\Delta=\sum_{i=1}^{n} \partial^{2} / \partial z_{i}^{2}$ is an $\mathrm{O}_{n}$-invariant differential operator. The commutator

$$
\left[\Delta, r^{2}\right]=2 \sum_{i=1}^{n}\left(z_{i} \frac{\partial}{\partial z_{i}}+\frac{\partial}{\partial z_{i}} z_{i}\right)=4 \sum_{i=1}^{n} z_{i} \frac{\partial}{\partial z_{i}}+2 n .
$$

Here $\sum_{i=1}^{n} z_{i} \partial / \partial z_{i}=E$ is Euler's degree operator. Thus $\Delta, r^{2}$ and $4 E+2 n$ together span a Lie algebra isomorphic to $\mathfrak{s l}_{2}$, which is the appropriate $\Gamma^{\prime}$ for this action. We see $\Gamma^{(2,0)}$ is spanned by $r^{2}, \Gamma^{(0,2)}$ by $\Delta$, and $\Gamma^{(1,1)}$ by $4 E+2 n$. In particular $\Gamma^{(1,1)}$ is abelian.

Now put $\mathscr{H}=\operatorname{ker} \Delta$. Thus $\mathscr{H}$ are the classical "spherical harmonics". Let $\mathscr{H}_{m}$ be the spherical harmonics of degree $m$. Then $4 E+2 n$ acts on $\mathscr{H}_{m}$ as the scalar operator $4 m+2 n$. Thus each $\mathscr{H}_{m}$ must be irreducible for $\mathrm{O}_{n}$ by Theorem 9(iii). Further, an easy computation shows that $\left(z_{1}+i z_{2}\right)^{m}$ is harmonic for all $n$, so $\mathscr{H}_{m} \neq\{0\}$. Specializing the rest of Theorem 9 to this case we have the following basic facts of the classical theory of spherical harmonics.

(i) The spherical harmonics $\mathscr{H}_{m}$ of each degree $n$ form an irreducible $\mathrm{O}_{n}$ module. The $\mathscr{H}_{m}$ are mutually distinct.

(ii) Every irreducible $\mathrm{O}_{n}$ submodule of polynomials is isomorphic to some $\mathscr{H}_{m}$.

(iii) Every polynomial may be written as a sum $\sum P_{i}\left(r^{2}\right) h_{i}$, where the $P_{i}$ are polynomials in one variable and the $h_{i}$ are harmonic. (In this case, the 
expression is easily seen to be unique.) More precisely, if $\mathscr{P}_{m}$ is the space of polynomials of degree $m$, then

$$
\mathscr{P}_{m} \simeq \bigoplus_{0 \leq 2 k \leq m} r^{2 k} \mathscr{H}_{m-2 k}
$$

is the decomposition of $\mathscr{P}_{m}$ into irreducible $\mathrm{O}_{n}$-modules.

(b) The Hodge decomposition theory. Let $V=V_{R}$ be a real vector space of dimension $2 n$. Let $J$ be a complex structure on $V$; that is, $J$ is an operator on $V$ such that $J^{2}=-1$. An inner product $($,$) on V$ is said to define a compatible Hermitian structure for $J$ if

$$
(J x, y)=-(x, J y) .
$$

Such inner products exist. Choose one and denote it by $($,$) . The form$ $\gamma(x, y)=(J x, y)$ is called the Kähler form attached to $J$ and to $($,$) . It is a$ symplectic form on $V$.

Now consider $V_{\mathbf{C}} \simeq V \otimes_{\mathbf{R}} \mathrm{C}$. We see that $V_{\mathbf{C}}=V^{+} \otimes V^{-}$where $V^{ \pm}$is the $\pm \sqrt{-1}$ eigenspace for $J$. Extend (, ) to a symmetric, complex bilinear form on $V_{\mathrm{C}}$. Then for $x, y \in V^{+}$, we compute

$$
i(x, y)=(i x, y)=(J x, y)=-(x, J y)=-(x, i y)=-i(x, y) .
$$

Hence $V^{+}$is isotropic for $($,$) . Similarly V^{-}$is isotropic for $($,$) . So V^{+}$ and $V^{-}$are in duality via $($,$) . Thus the subgroup of the isometry group of$ $\left(\right.$, ) preserving $V^{+}$and $V^{-}$is isomorphic by restriction to $\mathrm{GL}\left(V^{+}\right)$(or to $\left.\mathrm{GL}\left(V^{-}\right)\right)$.

We are interested in the relation between the Kähler form $\gamma$ and the action of $\mathrm{GL}\left(V^{+}\right)$on the exterior algebra $\Lambda\left(V_{\mathrm{C}}\right)$. We note the decomposition

$$
\Lambda\left(V_{\mathbf{C}}\right) \simeq \Lambda\left(V^{+}\right) \otimes \Lambda\left(V^{-}\right) \simeq \bigoplus_{0 \leq p, q \leq n} \Lambda^{p}\left(V^{+}\right) \otimes \Lambda^{q}\left(V^{-}\right) .
$$

Write $\Lambda^{p}\left(V^{+}\right) \otimes \Lambda^{q}\left(V^{-}\right)=\Lambda^{p, q}$. Evidently the $\Lambda^{p, q}$ are invariant under $\mathrm{GL}\left(V^{+}\right)$. In fact the partial degree operators $\mathrm{deg}^{+}$and $\mathrm{deg}^{-}$, which multiply $\Lambda^{p, q}$ by $p$ and $q$ respectively, obviously commute with the action of $\operatorname{GL}\left(V^{+}\right)$. We can get two other operators commuting with $\mathrm{GL}\left(V^{+}\right)$from the Kähler form $\gamma$. We note that $\gamma \in \Lambda^{2}\left(V_{\mathbf{C}}^{*}\right)$. However, by means of $\left(\right.$, ) we can identify $V_{\mathbf{C}}$ and $V_{\mathbf{C}}^{*}$, so that we may also consider that $\gamma \in \Lambda^{2}\left(V_{\mathbf{C}}\right)$. In fact, since $V^{ \pm}$are isotropic for $\gamma$, we will have $\gamma \in \Lambda^{1,1}$. Since $\gamma$ is clearly $\mathrm{GL}\left(V^{+}\right)$-invariant we can get two more operators commuting with $\mathrm{GL}\left(V^{+}\right)$. Namely, let $\Lambda$ be the operation of multiplication by $\gamma$ when we consider $\gamma \in \Lambda^{1,1}$. Let $L$ be the operation of inner multiplication, or contraction, with $\gamma$, when we consider $\gamma \in \Lambda^{2}\left(V_{\mathrm{C}}^{*}\right)$. It is not hard to compute that

$$
[L, \Lambda]=\mathrm{deg}^{+}+\mathrm{deg}^{-}-n
$$


so that $L, \Lambda, \operatorname{deg}^{+}+\operatorname{deg}^{-}-n$, and $\mathrm{deg}^{+}-\mathrm{deg}^{-}$together form a Lie algebra, isomorphic to $\mathrm{gl}_{2}$, with $\mathrm{deg}^{+}-\mathrm{deg}^{-}$the central operator, and the other three forming an $\mathrm{sl}_{2}$.

Referring back to Theorem 7, we recognize that we are here in the situation of $G=\mathrm{GL}\left(V^{+}\right), U=\{0\}$ and $W \simeq V^{+} \oplus V^{+*}$. We know therefore that the $\mathfrak{g} 1_{2}$ constructed above generates the full commuting algebra of $\operatorname{GL}\left(V^{+}\right)$. Taking into account the known structure of $\mathfrak{g l}_{2}$-modules [Se], and the particular form of $[L, \Lambda]$, we make the following assertions.

(i) Let ${ }^{\circ} \Lambda^{p, q}=(\operatorname{ker} L) \cap \Lambda^{p, q}$. Then $\Lambda^{k}:{ }^{\circ} \Lambda^{p, q} \rightarrow \Lambda^{p+k, q+k}$ is an isomorphism for $p+q+k \leq n$, and zero for $p+q+k>n$.

(ii) Put ${ }^{k} \Lambda^{p, q}=\Lambda^{k}\left({ }^{\circ} \Lambda^{p-k, q-k}\right)$. Then $\Lambda^{p, q}=\bigoplus_{k}{ }^{k} \Lambda^{p, q}$ and these ${ }^{k} \Lambda^{p, q}$ are irreducible mutually inequivalent modules for $\mathrm{Gl}\left(V^{+}\right)$.

(iii) In particular, each irreducible $\mathrm{GL}\left(V^{+}\right)$submodule of $\Lambda\left(V_{\mathrm{C}}\right)$ is isomorphic to ${ }^{\circ} \Lambda^{p, q}$ for unique $p$ and $q$.

The above facts, with $\mathrm{GL}\left(V^{+}\right)$suppressed, are usually referred to as the Hodge decomposition for forms. Chern [Ch] noticed that the Hodge decomposition was representation theoretic in character, and Serre [Se] pointed out that the operators $\Lambda$ and $L$ generated an $\mathfrak{s l}_{2}$. However, I do not know if the full duality has been recognized before.

Actually $\mathrm{GL}\left(V^{+}\right)$is not usually considered in this context. Rather, by virtue of the real structure underlying $V_{\mathrm{C}}$, one has on $V_{\mathrm{C}}$ a complex conjugation operator, which interchanges $V^{+}$and $V^{-}$. The subgroup $\operatorname{GL}\left(V^{+}\right)$commuting with complex conjugation is $\mathrm{U}$, the unitary group. The group $\mathrm{U}$ will also leave $V_{\mathbf{R}}$ invariant, and on $V_{\mathbf{R}}$ we may identify $U$ to the group leaving $($,$) fixed$ and commuting with $J$. Since the representation of $\mathrm{GL}\left(V^{+}\right)$on $\Lambda\left(V_{\mathrm{C}}\right)$ is holomorphic, the irreducible constituents will still be irreducible on restriction to the real form $U$. So the Hodge decomposition may also be regarded as a decomposition according to U-modules. In any case, the analogy with spherical harmonics is clear. The ${ }^{\circ} \Lambda^{p, q}$, analogs of harmonic polynomials, are generally referred to as primitive forms.

(c) The de Rham complex. For a vector space $V$, the algebra $\mathscr{A}\left(V^{*}, V^{*}\right) \simeq$ $S\left(V^{*}\right) \otimes \Lambda\left(V^{*}\right)$ is the algebra of polynomial-valued exterior forms on $V$, so we may term it the polynomial de Rham complex. We have the obvious action of $\mathrm{GL}\left(V^{*}\right)$ on it. In terms of $\S 3$, this is the case $G=\mathrm{GL}\left(V^{*}\right), U=W=V^{*}$. Here $\Gamma^{\prime}$ is four dimensional. It contains the exterior derivation operator $d$, given by

$$
d=\sum d x_{i} \frac{\partial}{\partial x_{i}}
$$

in coordinates, and the co-differentiation

$$
d^{*}=\sum x_{i} i\left(d x_{i}\right)
$$


where $i\left(d x_{i}\right)$ indicates a contraction or inner multiplication operator. A straightforward computation shows that $d^{2}=d^{* 2}=0$, and

$$
2\left\{d, d^{*}\right\}=\operatorname{deg}_{p}+\operatorname{deg}_{e},
$$

where $\operatorname{deg}_{p}$ is the polynomial degree, or Euler operator, and $\operatorname{deg}_{e}$ is the exterior degree operator. Thus on $S^{k}\left(V^{*}\right) \otimes \Lambda^{l}\left(V^{*}\right), \operatorname{deg}_{p}$ is multiplication by $k$ and $\operatorname{deg}_{e}$ is multiplication by $l$. Thus on the space $\bigoplus_{k+l=m} S^{k}\left(V^{*}\right) \otimes \Lambda^{l}\left(V^{*}\right)$, the space of forms of total degree $m$, we see $\operatorname{deg}_{p}+\operatorname{deg}_{e}$ acts as the scalar $m$. Thus the operator

$$
2\left(\frac{d d^{*}+d^{*} d}{\operatorname{deg}_{p}+\operatorname{deg}_{e}}\right)=1-P,
$$

where 1 is the identity and $P$ is projection onto the constants, which are $S^{\circ} \otimes \Lambda^{\circ}$. In other words, we have an explicit construction of a chain homotopy between 1 and $P$. The existence of such a homotopy is useful for homological algebra. For example, it is used in [J] as a basic step in the proof of the Hilbert Syzygy Theorem.

The explicit module structure of $\mathscr{A}\left(V^{*}, V^{*}\right)$ is fairly pretty. It is not hard to show that except for $S^{\circ} \otimes \Lambda^{\circ}$ all the irreducible submodules of $\mathscr{A}$ for the $\Gamma^{\prime}$ spanned by $d, d^{*}, \operatorname{deg}_{p}$ and $\operatorname{deg}_{e}$ are 2 dimensional. From this, one concludes each GL $\left(V^{*}\right)$-module occurs twice, once in $S^{l} \otimes \Lambda^{k}$ as the space of closed forms and once in $S^{l+1} \otimes \Lambda^{k-1}$ as the space of co-closed forms. The Poincare lemma follows. Using Young's diagrams, another product of Theorem 1A, as explained in [W], we may be very concrete about these modules. The Young's diagram for $S^{l}$ is

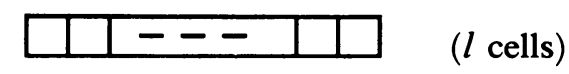

while the Young's diagram for $\Lambda^{k}$ is

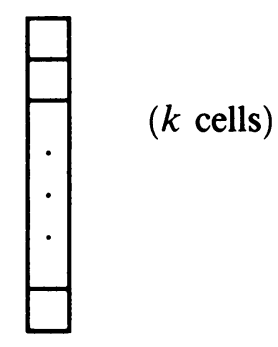

In forming the tensor product $S^{l} \otimes \Lambda^{k}$ these diagrams may be abutted in two 
ways. Diagrammatically, we may write

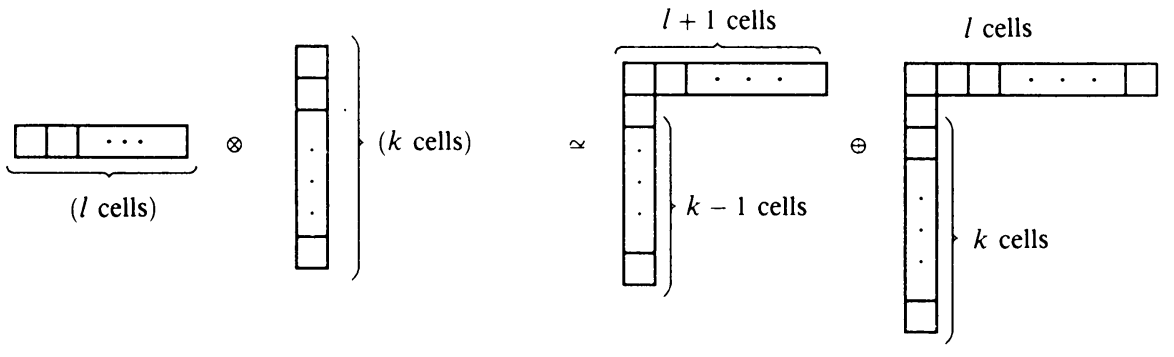

The third and fourth diagrams represent the closed and co-closed forms respectively. The Dolbeault complex may be handled in a parallel manner.

(d) Cohomology of the unitary group. In the cases $G=\mathrm{GL}(V)$ and the algebras $S\left(V^{k}\right)$ or $\Lambda\left(V^{k}\right)$-that is, when only covariant tensors of a given symmetry type occur-the situation is particularly simple. In this case, we may write $V^{k} \simeq V \otimes D$, where $D$ is an auxiliary vector space of dimension $k$. Thus $\mathrm{GL}(D)$ acts in an obvious way on $S(V \otimes D)$ or $\Lambda(V \otimes D)$, and Theorem 8 just says that $\mathfrak{g} 1(V)$ and $\mathfrak{g} 1(D)$ generate each other's commutant inside the appropriate Weyl or Clifford algebra. Thus $S(V \otimes D)$ breaks up into a sum of irreducible $\mathrm{GL}(V) \times \mathrm{GL}(D)$ modules, each occurring once, and there is established thereby a bijection between certain representations of $\mathrm{GL}(V)$ and certain representations of $\mathrm{GL}(D)$. The correspondence may be conveniently described in terms of Young's diagrams. Although the computations which justify this description are not difficult, we will leave them out.

The representations of $\mathrm{GL}(V)$ occurring in the full tensor algebra over $V$ may be described by Young's diagrams with at most $\operatorname{dim} V$ rows. Similarly, representations of $\mathrm{GL}(D)$ are described by diagrams with at most $\operatorname{dim} D$ rows. In the joint action on $S(V \otimes D)$ it may be seen that precisely those representations of $\mathrm{GL}(V)$ or $\mathrm{GL}(D)$ occur which have diagrams with at most $\min (\operatorname{dim} V, \operatorname{dim} D)$ rows. The only plausible possibility for matching is to match those representations of $\mathrm{GL}(V)$ and $\mathrm{GL}(D)$ which have the same Young's diagram, and this is indeed what happens.

The case of $\Lambda(V \otimes D)$ is more interesting. Here representations of $\mathrm{GL}(V)$ occur whose Young's diagrams have at most $\operatorname{dim} D$ columns. Similarly, representations of $\mathrm{GL}(D)$ occur whose Young's diagrams have at most $\operatorname{dim} V$ columns. A representation of $\mathrm{GL}(V)$ and a representation of $\mathrm{GL}(D)$ are matched if their Young's diagrams are obtained from each other by flipping them over the diagonal. Thus for example, representations with diagrams

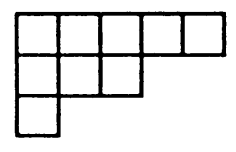

and

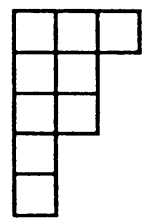

correspond. 
This circumstance may be used to compute the cohomology of the unitary group. As is well known [W] the cohomology of a compact Lie group is the algebra of invariants for the adjoint action in the exterior algebra of the Lie algebra of the group. For the unitary group, we may complexify and consider the action by conjugation of $\mathrm{GL}(V)$ on $\Lambda\left(V \otimes V^{*}\right)$. The action of $\mathrm{GL}(V)$ by conjugation results from the diagonal embedding of $\mathrm{GL}(V)$ into $\mathrm{GL}(V) \times$ $\mathrm{GL}(V)$ acting by left and right translations. The action of $\mathrm{GL}(V) \times \mathrm{GL}(V)$ decomposes into a direct sum of irreducible representations $\rho \otimes \sigma$, where $\rho$ is a representation of the first factor and $\sigma$ a representation of the second factor. The fixed points for the diagonal group will arise when $\rho=\sigma$. There will be one fixed vector for each such coincidence. From the above discussion, we know the pairs $\{\rho, \sigma\}$ which can possibly occur. There will be one coincidence $\rho=\sigma$ for each Young's diagram, of at most $n$ rows and $n$ columns, which is symmetric across the diagonal. Among such diagrams there is an obvious collection from which the others can be generated. These are best described by writing down the first several:

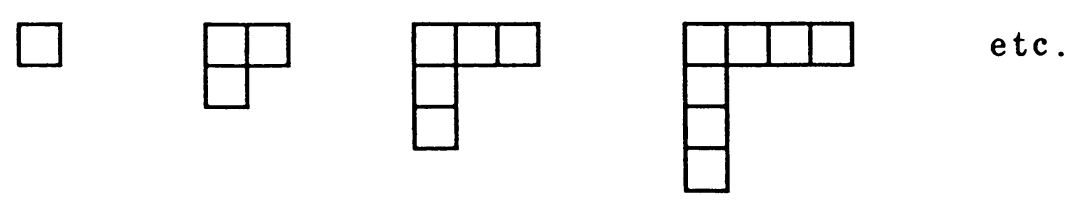

This description allows us immediately to write down the Poincare polynomial of $\mathrm{U}_{n}$. A closer look would reveal that this description also yields the multiplicative structure of the cohomology, the above diagrams corresponding to the so-called "primitive generators".

One can give a similar description of the cohomology of the orthogonal and symplectic groups. That is somewhat trickier, but follows from considerations like those in topic (e) below. One can also calculate cohomology of Grassmann varieties in this manner.

(e) Symmetric invariants for classical Lie algebras. We can compute the invariant polynomials for the adjoint action of the classical groups. For $\operatorname{GL}(V)$, considerations as for cohomology yield the result easily. For $\mathrm{O}$ and $\mathrm{Sp}$ a certain amount of exercising with Young's diagrams is necessary, and this derivation is probably more difficult than better known ones. It does seem to have some interest, however, so we give it. We will use $\mathrm{Sp}$ as our example.

First, let us note that in principle Theorems $1 \mathrm{~A}$ and $1 \mathrm{~B}$ would permit computation of the invariants for any action of a classical group. Indeed, let the classical $G$ act on $V$ by an action. Then $V$ may be broken up into irreducible components and its symmetric algebra (for example) may be expressed as a sum of tensor products of the symmetric powers of the irreducible summands. Each 
of these summands may then be embedded somewhere in the mixed tensor algebra of the standard module. Given such an embedding, Theorems 1A and $1 \mathrm{~B}$ allow computation of the invariants in such a summand. Transferring this information back to $S(V)$ and putting it all together, you get a description of the polynomial invariants on $V$. Of course this principle is a very abstract one, for the individual steps in the above process can be very difficult. In particular, embedding the symmetric powers of even one irreducible module into the tensor algebra in an explicit way will be very difficult. However, precisely the above scheme yielded our basic Theorem 2, and it may also be applied in the case at hand.

Let the vector space $V$ be given a symplectic form and let $\mathrm{Sp}$ be the isometry group of this form. The adjoint action of $\mathrm{Sp}$ on its Lie algebra is isomorphic to the action on $S^{2}(V)$. The Young's diagram of $S^{2}$ is just $\square \square$. We want to compute $S^{n}\left(S^{2}(V)\right)$ as a subspace of the tensor algebra of $V$.

Proposition 1. As a $\mathrm{GL}(V)$-module, $S^{n}\left(S^{2}(V)\right)$ breaks up into a sum of one copy each of representations corresponding to Young's diagrams of $2 n$ cells and an even number of cells in each row.

Proof. Let $S_{2 n}$ be the symmetric group on the numbers $\{1,2, \ldots, 2 n\}$. Partition these numbers into $n$ pairs $\{2 i-1,2 i\}$ for $1 \leq i \leq n$. Let $B_{n}$ be the subgroup of $S_{2 n}$ which preserves this partition. According to the calculus of Young's diagrams, the GL(V)-modules in $S^{n}\left(S^{2}(V)\right)$ have the same Young's diagrams as the $S_{2 n}$-modules occurring in the permutation representation of $S_{2 n}$ on $S_{2 n} / B_{n}$. The intertwining algebra for this representation is the Hecke algebra $\mathscr{H}\left(S_{2 n} / / B_{n}\right)$ of $B_{n}$ double cosets in $S_{2 n}$, with convolution for multiplication.

Let us compute the $B_{n}$ double cosets in $S_{2 n}$. Take any $g \in S_{2 n}$. Then $g$ will take the pairs $\{2 i-1,2 i\}$ to some other collection of pairs. Let $\left\{a_{1}, a_{2}\right\}$ be a pair of the first partition. Then in the second partition, there will be a pair $\left\{a_{2}, a_{3}\right\}$. Possibly $a_{3}=a_{1}$ but likely not. Thus in the first partition there will be a pair $\left\{a_{3}, a_{4}\right\}$. We can continue in this fashion linking pairs by alternating between partitions until $a_{1}$ finally reappears in a pair of the second partition. This will complete the chain and we will have to start over again. By operating front and back with $B_{n}$ we see we can change the individual numbers in a given chain at will. What we cannot change is the lengths of the chains. These lengths form a partition of the number $n$. Thus the double coset space $B_{n} \backslash S_{2 n} / B_{n}$ is in natural correspondence with the partitions of $n$. The partitions of $n$ also parametrize the conjugacy classes in $S_{n}$. In fact, if we embed $S_{n}$ into $S_{2 n}$ by letting $S_{n}$ permute all the even numbers, then we see we have a direct correspondence between $B_{n}$ double cosets and conjugacy classes in $S_{n}$. Since each conjugacy class in $S_{n}$ is self-inverse, each $B_{n}$ double coset in $S_{2 n}$ is 
also self-inverse. Therefore the Hecke algebra $\mathscr{H}\left(S_{2 n} / / B_{n}\right)$ is commutative. ${ }^{4}$ Thus the representation of $S_{2 n}$ acting on $L^{2}\left(S_{2 n} / B_{n}\right)$ decomposes simply, each irreducible component occurring only once. Since the representations we claim occur are also clearly parametrized by partitions of $n$, to complete the proof it remains only to show that the desired representations actually do occur.

To demonstrate occurrence, it is simplest to return to the algebra $S\left(S^{2}(V)\right)$ and exhibit highest weight vectors corresponding to the desired $\mathrm{GL}(V)$-modules. (To produce these highest weight vectors is also of interest in its own right.) This is most easily done by introducing coordinates on $V$, which will give rise to coordinates $\left\{x_{i j}=x_{j i}: 1 \leq i, j \leq \operatorname{dim} V\right\}$ on $S^{2}(V)$. Given these coordinates, it is easy to check that the determinants

$$
\delta_{1}=x_{11}, \quad \delta_{2}=\operatorname{det}\left|\begin{array}{ll}
x_{11} & x_{12} \\
x_{12} & x_{22}
\end{array}\right|, \quad \delta_{3}=\operatorname{det}\left|\begin{array}{lll}
x_{11} & x_{12} & x_{13} \\
x_{12} & x_{22} & x_{23} \\
x_{13} & x_{23} & x_{33}
\end{array}\right|, \quad \text { etc. }
$$

are highest weight vectors for $\operatorname{GL}(V)$, and that monomials in the $\delta_{i}$ provide highest weights of all the desired kinds. This concludes the proof of Proposition 1 .

Next, we consider the Sp invariants. According to Theorem $1 \mathrm{~B}$, the $\mathrm{Sp}$ invariants on $\otimes^{2 n} V$ are all obtained by applying permutations on the factors to the particular invariant $\theta_{2 n}$. Let $s$ again denote the signum character of $S_{2 n}$. We observe that $\theta_{2 n}$ is actually an eigenvector for $B_{n}$, with eigencharacter the restriction of $s$ to $B_{n}$. Thus under $S_{2 n}$, we see $\theta_{2 n}$ generates (a quotient of) the induced representation

$$
\operatorname{ind}_{B_{n}}^{S_{2 n}} s \simeq s \otimes\left(\operatorname{ind}_{B_{n}}^{S_{2 n}} 1\right) \simeq s \otimes L^{2}\left(S_{2 n} / B_{n}\right) .
$$

Since tensor product with $s$ has the effect of flipping a Young's diagram over its diagonal, we see that the Young's diagrams which contribute to the $S_{2 n}$ module generated by $\theta_{2 n}$ are diagrams with an even number of elements in each column, at most one each.

On the other hand, we know that $\theta_{2 n} \in S^{n}\left(\Lambda^{2}(V)\right)$. Since $\theta_{2}$ generates a (Zariski) open and dense $\mathrm{GL}(V)$ orbit in $\Lambda^{2}$, we see that the $\mathrm{GL}(V)$-module generated by $\theta_{2 n}$ must span $S^{n}\left(\Lambda^{2}(V)\right)$. Combining Proposition 1 and the preceding paragraph, we may assert:

Proposition 2. As a $\mathrm{GL}(V)$-module, $S^{n}\left(\Lambda^{2}(V)\right)$ breaks up into a sum of one copy each of representations corresponding to Young's diagrams of $2 n$ cells with an even number of cells in each column.

Since $\theta_{2 n}$ generates $S^{n}\left(\Lambda^{2}(V)\right)$, we see every possible Young's diagram contributes to the $S_{2 n}$-module it generates. Therefore, we may conclude that each irreducible $\mathrm{GL}(V)$ submodule of $\otimes^{2 n} V$ of $\otimes^{2 n} V$ given by a Young's diagram

\footnotetext{
${ }^{4}$ This fact was told to me a long time ago by N. Iwahori, and this analysis was prompted by questions he raised.
} 
with columns of even length contains precisely one Sp invariant. In particular there is one Sp invariant for each Young's diagram consisting of two columns of even length. These of course give the well-known "basic invariants" which are easy to write down. Since they are obviously algebraically independent, a counting argument shows they generate all invariants.

(f) The Capelli identities. For our last application of the results of $\S 3$, we will try to provide insight into the mechanics of Weyl's treatment of classical invariant theory. The crux of his proofs is the Capelli identity, which is a fairly complicated object in itself. As we will see, this identity essentially describes the action of the center of the universal enveloping algebra of $\mathrm{GL}(V)$ on $\mathscr{A}\left(V^{k},\{0\}\right)$. Therefore we begin the discussion with some general remarks about how the center of the universal enveloping algebras behave under duality.

Consider $\mathscr{A}(U, W)$, where either $U$ or $W$ is trivial, so that actually $\mathscr{A}=$ $S(U)$ or $\mathscr{A}=\Lambda(W)$. In this case $\mathfrak{g r}=\mathfrak{g r}_{0}=\mathfrak{g}$ is either $\mathfrak{s p}(U \otimes \widetilde{U})$ or $\mathfrak{o}(W \otimes \widetilde{W})$. Further, a classical dual pair in $\mathfrak{g}$ consists of a pair $\left(\Gamma, \Gamma^{\prime}\right)$ of classical Lie algebras. We will give notation for various objects associated to $\Gamma$; notation for $\Gamma^{\prime}$ will be parallel. Let $A(\Gamma)$ be the subalgebra of End $^{\circ}$ generated by $\Gamma$. Let $G$ be the classical group whose Lie algebra is $\Gamma$. We may think of $G$ as acting by automorphisms on $\mathrm{End}^{\circ}$. Then $A\left(\Gamma^{\prime}\right)$ is the algebra of invariants for $G$.

Let $\mathscr{U}(\Gamma)$ be the universal enveloping algebra of $\Gamma$ considered as an abstract Lie algebra. The inclusion of $\Gamma$ in End $^{\circ}$ as a Lie subalgebra defines a homomorphism $\alpha: \mathscr{U}(\Gamma) \rightarrow A(\Gamma)$. Let $\mathscr{Z}(\Gamma)$ be the invariants in $\mathscr{U}(\Gamma)$ under the adjoint action of $G$. (If $G=\mathrm{GL}_{n}, \mathrm{Sp}_{2 n}$, or $\mathrm{O}_{2 n+1}$, then $\mathscr{Z}(\Gamma)$ is just the center of $\mathscr{U}(\Gamma)$, but if $G=\mathrm{O}_{2 n}$, it is properly inside the center.) Since $G$ acts reductively on $\mathscr{U}(\Gamma)$, the image of $\mathscr{Z}(\Gamma)$ under $\alpha$ is precisely the space of $G$ invariants in $A(\Gamma)$, that is

$$
\alpha: \mathscr{Z}(\Gamma) \rightarrow A(\Gamma) \cap A\left(\Gamma^{\prime}\right)
$$

is surjective. Similarly $\alpha^{\prime}$ is surjective from $\mathscr{Z}\left(\Gamma^{\prime}\right)$ to $A(\Gamma) \cap A\left(\Gamma^{\prime}\right)$. Thus $\alpha(\mathscr{Z}(\Gamma))=\alpha^{\prime}\left(\mathscr{Z}\left(\Gamma^{\prime}\right)\right)=A(\Gamma) \cap A\left(\Gamma^{\prime}\right)$. This equality enforces a certain correspondence between characters of $\mathscr{Z}(\Gamma)$ and of $\mathscr{Z}\left(\Gamma^{\prime}\right)$ arising from submodules of $\mathscr{A}(U, W)$. This correspondence must clearly be compatible with the correspondence of Theorem 8. In fact, each determines the other.

Now consider the Capelli identity. We will first state it in its simplest form. Consider a matrix $\left\{A_{i j}: 1 \leq i, j \leq n\right\}$ of noncommuting variables. By the determinant of this matrix, we mean the sum

$$
\sum_{g \in S_{n}} s(g) A_{g(1) 1} A_{g(2) 2} \cdots A_{g(n) n} .
$$

Thus we take one element from each column, multiply together keeping the order indicated by the columns, and sum with appropriate signs. Thus for 
example

$$
\operatorname{det}\left(\begin{array}{l}
A_{1} A_{1} \\
A_{2} A_{2}
\end{array}\right)=A_{1} A_{2}-A_{2} A_{1} .
$$

Now consider the $n \times m$ matrices $M_{n, m}(\mathbf{C})$. Let $\mathrm{GL}_{n}$ act on $M_{n, m}$ by multiplication on the left. The "polarization" operators $\pi_{k l}^{\prime}, 1 \leq k, l \leq m$, are defined by

$$
\pi_{k l}^{\prime}=\sum_{i=1}^{n} x_{i k} \frac{\partial}{\partial x_{i l}} .
$$

These operators commute with $\mathrm{GL}_{n}$, and in fact they form the dual algebra $\Gamma^{\prime}$ for it, which in this case is simply the (Lie algebra of) $\mathrm{GL}_{m}$ acting by right multiplication. The basic Capelli identity says

$$
\operatorname{det}\left(\pi_{k l}^{\prime}+\delta_{k l}(m-k)\right)=\sum \operatorname{det}\left(x_{i_{a} j_{b}}\right) \operatorname{det}\left(\frac{\partial}{\partial x_{i_{a} j_{b}}}\right),
$$

where the sum is over all pairs of $m$-tuples $1 \leq i_{1}<i_{2}<\cdots<i_{m} \leq n$. Thus in particular, if $m>n$ the left-hand side is zero, and if $n=m$ it is equal to

$$
\Omega=\operatorname{det}\left(x_{i j}\right) \operatorname{det}\left(\frac{\partial}{\partial x_{i j}}\right) \text {. }
$$

This is known as the Cayley $\Omega$-process [W].

In any case, the right-hand side of $(C)$ is visibly invariant under conjugation by $\mathrm{GL}_{m}$, while the left-hand side, being formed from polarizations, commutes with $\mathrm{GL}_{n}$. In terms of the general discussion above, we see that the Capelli identity gives us an explicit construction of elements of $A(\Gamma) \cap A\left(\Gamma^{\prime}\right)$ for this particular pair. It is interesting that from knowledge of these elements, it is possible to recover the complete invariant theory.

To prove equation $(\mathrm{C})$, we take our cue from the interpretation just given, and commence by showing the left-hand side, considered as an element of $\mathscr{U}\left(\mathfrak{g} 1_{m}\right)$, is central. For this we need only the commutation relations in $\mathfrak{g} 1_{m}$, not the specific form of the operators.

First note some formal properties of $\operatorname{det}\left\{A_{i j}\right\}$ for arbitrary $A_{i j}$. From the definition, it is clear that $\operatorname{det}\left\{A_{i j}\right\}$ is antisymmetric in its rows, just as normal determinants are. Thus, the usual column expansion is good for the first column. Also various expansions relating to partitioning of the columns are valid, so long as the columns are kept in their proper order. In particular, take the $i$ th column, and partition the columns into the set preceding $i$, the set following $i+1$, and the pair $\{i, i+1\}$. Then we have

$$
\operatorname{det}\left\{A_{j k}\right\}=\sum_{l<m}\left(\sum_{\alpha} B_{\alpha} \operatorname{det}\left(\begin{array}{cc}
A_{l i} & A_{l i+1} \\
A_{m i} & A_{m i+1}
\end{array}\right) C_{\alpha}\right),
$$

where $\alpha$ runs over all $(i-1)$ tuples of rows not containing the $l$ th or $m$ th, and $B_{\alpha}$ is the determinant of the matrix defined by these rows and the first $i-1$ columns, and $C_{\alpha}$ is the determinant of the matrix determined by the 
remaining $n-i-1$ rows and columns. This formula allows us to study the effect of reversing two columns. We note the pleasant identity

$$
\operatorname{det}\left(\begin{array}{ll}
A & B \\
C & D
\end{array}\right)+\operatorname{det}\left(\begin{array}{ll}
B & A \\
D & C
\end{array}\right)=\operatorname{det}\left(\begin{array}{ll}
A & A \\
D & D
\end{array}\right)+\operatorname{det}\left(\begin{array}{ll}
B & B \\
C & C
\end{array}\right) .
$$

Thus we may say: the sum of the determinants of $\left\{A_{j r}\right\}$ and the matrix obtained by interchanging two adjacent columns is equal to the sum of the determinants of the two matrices obtained from $\left\{A_{j k}\right\}$ by putting both columns equal to one of them.

The commutator relations of the $\pi_{i j}^{\prime}$ are

$$
\left[\pi_{i j}^{\prime}, \pi_{k l}^{\prime}\right]=\delta_{j k} \pi_{i l}^{\prime}-\delta_{l i} \pi_{k j}^{\prime} .
$$

Denote the matrix whose determinant is the left-hand side of $(C)$ by $\Pi$. From these commutator relations one sees that all the $2 \times 2$ matrices formable from two copies of column $i$ of $\Pi$ have zero determinant except the matrices

$$
\pm\left(\begin{array}{cc}
\pi_{i i}^{\prime}+n-i & \pi_{i i}^{\prime}+n-i \\
\pi_{j i}^{\prime} & \pi_{j i}^{\prime}
\end{array}\right), \quad 1 \leq j \leq n, j \neq i,
$$

which have determinants $\pi_{i j}^{\prime}$. Thus the matrix obtained from $\Pi$ by replacing column $i+1$ with a second copy of column $i$ has the same determinant as the matrix obtained from $\Pi$ by replacing column $i+1$ by a column which is zero except for 1 in the $i$ th row.

Consider now the effect on det $\Pi$ of the adjoint action of the permutation matrix $\sigma_{i}$ which transposes $i$ and $i+1$. Because the diagonal entries of $\Pi$ are $\pi_{i i}^{\prime}+n-i$ and not simply $\pi_{i i}^{\prime}$ the action of $\sigma_{i}$ is not simply to transpose the $i$ th row and column of $\Pi$. A computation which is easier to perform than to describe succinctly shows that the actual effect of $\sigma_{i}$ on $\Pi$ is just right to cancel out the noncommutativity of columns $i$ and $i+1$ as described in the preceding paragraph. Thus $\Pi$ is actually invariant under $\sigma_{i}$, hence under the whole permutation group.

To finish the proof that $\operatorname{det} \Pi$ is central in $\mathscr{U}\left(\mathfrak{g} 1_{m}\right)$, it suffices to show that it commutes with any given $\pi_{i j}^{\prime}$, with $i \neq j$. We select $\pi_{n-1 n}^{\prime}$. Another formal property of our determinant is that $\left[B, \operatorname{det}\left\{A_{i j}\right\}\right]$ is the sum of the determinants of the matrices obtained from $\left\{A_{i j}\right\}$ by selecting some column and replacing each element in the column by its commutator with $B$. For each column except the $(n-1)$ th, taking commutators with $\pi_{n-1 n}^{\prime}$ kills everything but the bottom entry $\pi_{n j}^{\prime}$, which gets replaced with $\pi_{n-1 j}^{\prime}$. In the $(n-1)$ th column $\pi_{j n-1}^{\prime}$ is replaced with $-\pi_{j n}^{\prime}$, except for $\pi_{n n-1}^{\prime}$ which gets replaced with $\pi_{n-1 n-1}^{\prime}-\pi_{n n}^{\prime}$, and $\pi_{n-1 n-1}^{\prime}+1$, which gets replaced with $-\pi_{n-1 n}^{\prime}$. Using linearity in the $(n-1)$ th column, then in the $n$th row, we see that these $n$ determinants can be combined to form three determinants, one of a matrix gotten from $\Pi$ by replacing the $n$th row with a copy of the $(n-1)$ th row, one gotten by replacing the $(n-1)$ th column by the negative of the $n$th column, and the third gotten by replacing the $(n-1)$ th column by zeros except for -1 in the 
$n$th row. The determinant with the repeated row vanishes trivially. According to our remarks above, the remaining two determinants cancel each other, so the net result is zero. Thus det $\Pi$ is indeed central in $\mathscr{U}\left(\mathfrak{g} 1_{m}\right)$.

We can now prove (C). In fact, we will give an explicit description of $A\left(\mathfrak{g} 1_{n}\right) \cap$ $A\left(\mathfrak{g} 1_{m}\right)$.

Proposition 3. Let

$$
\pi_{j k}^{\prime}=\sum_{i=1}^{n} x_{i j} \frac{\partial}{\partial x_{i k}} \text { and } \pi_{j k}=\sum_{i=1}^{m} x_{j i} \frac{\partial}{\partial x_{k i}}
$$

be the standard basis elements for $\Gamma^{\prime}=\mathfrak{g} 1_{m}$ and $\Gamma=\mathfrak{g} 1_{n}$ respectively. Then $A(\Gamma) \cap A\left(\Gamma^{\prime}\right)$ is a polynomial algebra on the generators

$$
\begin{aligned}
\gamma_{l} & =\sum_{\alpha} \operatorname{det}\left\{\pi_{\alpha_{j} \alpha_{k}}+\delta_{j k}(l-j)\right\}=\sum_{\beta} \operatorname{det}\left\{\pi_{\beta_{j} \beta_{k}}^{\prime}+\delta_{j k}(l-j)\right\} \\
& =\sum_{\alpha, \beta} \operatorname{det}\left(x_{\alpha_{j} \beta_{k}}\right) \operatorname{det}\left(\frac{\partial}{\partial x_{\alpha_{j} \beta_{k}}}\right) .
\end{aligned}
$$

Here $\alpha$ runs over all l-tuples from the numbers 1 through $n$ and $\beta$ runs through $l$-tuples of the numbers 1 through $m$. The operator $\gamma_{l}$ is zero if $l>\min (m, n)$. Proof. We have shown that the first expression for $\gamma_{l}$ is in the center of $A(\Gamma)$, hence in $A(\Gamma) \cap A\left(\Gamma^{\prime}\right)$. Similarly for the second expression. The third is easily shown to be in $A(\Gamma) \cap A\left(\Gamma^{\prime}\right)$ by an argument like that given in $\S 4(\mathrm{~d})$, and in this form the $\gamma_{l}$ are obviously the classically known free generators for $A(\Gamma) \cap A\left(\Gamma^{\prime}\right)$. To finish, we need to show that, say, the first expression equals the third.

To do this, the most direct method seems to be to write down all the joint highest weight vectors for the action of $\mathrm{Gl}_{n} \times \mathrm{GL}_{m}$ on $M_{n, m}$ and verify that the two operators do the same to each. This is actually not too hard to do. Let

$$
\delta_{k}=\operatorname{det}\left\{x_{i j}\right\} \text { for } 1 \leq i, j \leq k .
$$

Then the highest weight vectors for $\mathrm{GL}_{n} \times \mathrm{GL}_{m}$ have the form [Z] $\Delta\left(p_{i}\right)=$ $\delta_{1}^{p_{1}} \delta_{2}^{p_{2}} \cdots \delta_{q}^{p_{q}}$ for arbitrary nonnegative integers $p_{i}$. This assertion is fairly easy to verify. One simply computes to show they are joint highest weight vectors, then observes that the modules they represent exhaust all possible isotypes.

It remains to show that the actions of the various expressions for $\gamma_{l}$ agree on the $\Delta\left(p_{i}\right)$. By an inductive argument, the main point to verify is that all expressions for $\gamma_{l}$ kill $\Delta\left(p_{i}\right)$ if $p_{i}=0$ for $i \geq l$. For the third expression for the $\gamma_{l}$ this is clear. For the first two expressions, the crucial observation is that since the $\Delta\left(p_{i}\right)$ are highest weight vectors, i.e., $\pi_{j k}\left(\Delta\left(p_{i}\right)\right)=0=\pi_{j k}^{\prime}\left(\Delta\left(p_{i}\right)\right)$ if $j<k$, we have the equation

$$
\operatorname{det}\left\{\pi_{\alpha_{j} \alpha_{k}}+\delta_{j k}(l-j)\right\}\left(\Delta\left(p_{i}\right)\right)=\left(\prod_{j=1}^{l}\left(\pi_{\alpha_{j} \alpha_{j}}+l-j\right)\right)\left(\Delta\left(p_{i}\right)\right) .
$$


The last factor in $\prod_{j=1}^{l}\left(\pi_{\alpha_{j} \alpha_{j}}+l-j\right)$ is $\pi_{\alpha_{l} \alpha_{l}}$. Since $\alpha_{l} \geq l$, this will kill any expression which involves only $x_{i j}$ with $i, j<l$, as is the case with $\Delta\left(p_{i}\right)$ if $p_{i}=0$ for $i \geq l$. This concludes Proposition 3 .

There is an analogue of Proposition 3 for the pair $(\mathfrak{s p}, \mathfrak{o})$. We leave its details as an exercise for the reader.

\section{CONCLUDING REMARKS}

(a) The theory of reductive dual pairs also makes sense in a transcendental context, that of the oscillator representation of symplectic groups over local fields. In that context, one considers a pair $\left(G, G^{\prime}\right)$ of reductive subgroups which are mutual centralizers in $\mathrm{Sp}$. When the field is $\mathbf{R}$, and one of $G$ or $G^{\prime}$ is compact, the theory presented here may be applied directly to obtain a duality between certain unitary representations of $G$ and of $G^{\prime}$. If $G$ is compact and $G^{\prime}$ is not, then $G^{\prime}$ will always have a Hermitian symmetric structure, and the representations of $G^{\prime}$ will be of holomorphic type, i.e., will have highest weights. Essentially this line of attack has been followed by several authors [Ge, G-K, $\mathrm{K}-\mathrm{V}, \mathrm{Sa}$ ] to obtain examples in various cases of holomorphic representations.

When neither $G$ nor $G^{\prime}$ is compact, essentially the same duality phenomenon occurs, but the situation is technically more complicated, and the algebraic results given here do not apply directly. However, it should be noted that the homomorphism of $\S 4(f)$ between centers of enveloping algebras of $\Gamma$ and $\Gamma^{\prime}$ still exists and controls the infinitesimal characters of corresponding representations. This information on infinitesimal characters, along with some qualitative features, should essentially determine the duality correspondence.

(b) In his book, Weyl discusses two Main Theorems of Classical Invariant Theory. In this paper we have been exclusively concerned with the First Main Theorem, a list of generators for the ring of invariants. The Second Main Theorem describes the relations between these invariants. In a recent discussion with Michéle Vergne, a use for the Second Main Theorem was proposed. Consider a reductive dual pair $\left(G, G^{\prime}\right)$ in $\operatorname{Sp}_{2 n}(\mathbf{R})$, with $G$ compact and $G^{\prime}$ noncompact. By means of Theorem 9, one can embed the representations of $G^{\prime}$ occurring in the restriction of the oscillator representation in certain spaces of holomorphic functions on the symmetric space of $G^{\prime}$. These embeddings are not in general surjective, so there is a problem to describe their range. Using the Second Main Theorem, one can write down some additional differential equations (i.e., additional to the Cauchy-Riemann equations) satisfied by functions in the range of such an embedding. These systems should be fairly interesting examples for P.D.E. fans.

Conversely, the representation-theoretic point of view provides insight into the varieties defined by the Second Main Theorem. In the notation of Theorem 9 , these varieties must just be the orbits of $G^{(1,1)}$ acting on $\Gamma^{(0,2)}$. 
The results in this paper have been several years in formation. I am indebted to many people for helpful conversations or other stimulation. I would like to mention in particular Steve Gelbart and Howard Garland.

\section{POSTSCRIPT}

When writing this paper, the author hoped that the viewpoint it took, and the general Theorems 7, 8, 9 were new. On the other hand, the examples (a) through ( $\mathrm{f}$ ) of $\S 4$ were understood to be individually well known, and the point was to discuss them from the uniform viewpoint provided by $\S 3$. In the years since the paper was written, the author has learned much more precisely how its contents connect with specific parts of the literature. We summarize here some of these connections. The book [L] has many formal identities, some of which can be seen to be equivalent to various of the decompositions of the algebras $\mathscr{A}(U, V)$ described in Theorems 8 and 9 . In particular, the decomposition of the polynomials on the $n \times n$ matrices under the action of $\mathrm{GL}_{n} \times \mathrm{GL}_{m}$ is equivalent to "Cauchy's Lemma", a 19th century identity. Weyl [W, pp. 202207] makes use of this identity without seeming to be conscious of its geometric import. The book of Zhelobenko [Z] treats the action of $\mathrm{GL}_{n} \times \mathrm{GL}_{m}$ on the $n \times m$ matrices in a similar spirit to this paper, and gives formulas for the highest weight vectors, from which our formulas in $\S 4(\mathrm{f})$ derive. Seshadri [Sh] espouses a point of view toward the Capelli identities similar to ours. The "polynomial de Rham complex" is widely known as the Koszul complex. Several papers of Kostant treat several of the examples of $\S 4$, and also some of the machinery of $\S \S 1,2$, sometimes from a similar point of view [Ks1, Ks2, Ks3]. In particular, the descriptions of $S^{n}\left(S^{2}(V)\right)$ and $S^{n}\left(\Lambda^{2}(V)\right)$ of $\S 4(\mathrm{e})$ are examples of his result about actions of Levi components on enveloping algebras of nilradicals. They also are examples of Helgason's theorem on representations with $K$-fixed vectors [He]. An early reference for $S^{n}\left(S^{2}(V)\right)$ is [Th]. Finally, several special cases of Theorems 7, 8, 9 have appeared in the physics literature, some before and some after this paper was written. See [Hol] for some references.

\section{REFERENCES}

[ABP] M. Atiyah, R. Bott and V. Patodi, On the heat equation and the Index Theorem, Invent. Math. 19 (1973), 279-330.

[Ch] S. S. Chern, On a generalization of Kähler geometry, Algebraic Geometry and Topology, A Symposium in Honor of S. Lefschetz, Princeton Univ. Press, Princeton, N.J., 1975.

[Ge] S. Gelbart, Holomorphic discrete series for the real symplectic group, Invent. Math. 19 (1973), 49-58.

[G-K] K. Gross and R. Kunze, Bessel functions and representation theory. I, II, J. Funct. Anal. 25 (1977), 1-49.

[He] S. Helgason, $A$ duality for symmetric spaces with applications to group representations, Adv. in Math. 5 (1970), 1-54.

[Ho1] R. Howe, Dual pairs in physics: Harmonic oscillators, photons, electrons, and singletons, Lectures in Appl. Math., vol. 21, Amer. Math. Soc., Providence, R.I., 1985, pp. 179-206.

[J] N. Jacobson, Basic algebra. II, Freeman, New York, 1980. 
[K] V. Kac, Simple irreducible graded Lie algebras of finite growth, Izv. Akad. Nauk SSSR 32 (1968), 1323-1367; English transl., Math. USSR-Izv. 2 (1968), 1271-1311.

[K-V] N. Kashiwara and M. Vergne, On the Segal-Shale-Weil representations and harmonic polynomials, Invent. Math. 44 (1978), 1-47.

[Ks1] B. Kostant, Graded manifolds, graded Lie theory, and prequantization, Differential Geometrical Methods in Mathematical Physics (Proc. Sympos. Univ. Bonn, Bonn, 1975), Lecture Notes in Math., vol. 570, Springer-Verlag, Berlin, 1977, pp. 177-306.

[Ks2] __ Lie algebra cohomology and the generalized Borel-Weil Theorem, Ann. of Math. (2) 74 (1961), 324-387.

[Ks3] _ _ A theorem of Frobenius, a theorem of Amitsur-Levitski and cohomology theory, J. Math. Mech. 7 (1958), 237-264.

[L] D. Littlewood, The theory of group characters and matrix representations of groups, Oxford Univ. Press, Oxford, 1940.

[Sa] M. Saito, Représentations unitaires des groupes symplectiques, J. Math. Soc. Japan 24 (1972), 232-251.

[Se] J.-P. Serre, Algèbres de Lie semi-simples complexes, Benjamin, Reading, Mass., 1966.

[Sh] C. Seshadri, Letter to M. F. Atiyah.

[Sh] D. Shale, Linear symmetries of free boson fields, Trans. Amer. Math. Soc. 103 (1962), 149-167.

[Th] R. Thrall, On symmetrized Kronecker powers and the structure of the free Lie ring, Amer. J. Math. 64 (1942), 371-388.

[H] T. Tilgner, (a) Graded generalizations of Weyl and Clifford algebras, J. Pure Appl. Algebra 10 (1977), 163-168.

(b) A graded generalization of Lie triples, J. Algebra 47 (1977), 190-196.

[Wi] A. Weil, Introduction a l'étude des variétés Kähleriennes, Hermann, Paris, 1958.

[Wi2] __, Sur certains groupes d'opérateurs unitaires, Acta Math. 11 (1964), 143-211.

[W] H. Weyl, The classical groups, Princeton Univ. Press, Princeton, N.J., 1946.

[Z.] D. Zhelobenko, Compact Lie groups and their representations, Transl. Math. Mono., no. 40, Amer. Math. Soc., Providence, R.I., 1973.

Department of Mathematics, Yale University, New Haven, Connecticut 06520 